\title{
Emergency collision avoidance control strategy based on four-wheel steering and differential braking
}

\author{
Haiqing $\mathrm{Li}^{\mathrm{a} *}$, Yongfu Li ${ }^{\mathrm{b}}$, Taixiong Zheng ${ }^{\mathrm{a}}$, Jiufei $\mathrm{Luo}^{\mathrm{a}}$ and Zonghuan $\mathrm{Guo}^{\mathrm{c}}$ \\ ${ }^{a}$ School of Advanced Manufacturing Engineering, Chongqing University of Posts and \\ Telecommunications, Chongqing, China;
}

${ }^{b}$ Key Laboratory of Intelligent Air-Ground Cooperative Control for Universities in Chongqing, College of Automation, Chongqing University of Posts and Telecommunications, Chongqing, China;

${ }^{c}$ College of Automation, Chongqing University, Chongqing, China;

Haiqing Li (Corresponding author) received his Ph.D. degree from Nanjing University of Aeronautics and Astronautics, China, in 2019. He is currently a lecturer in Chongqing University of Posts and Telecommunications, China. His research interests include vehicle dynamics control and intelligent vehicle control.

E-mail: lihq@cqupt.edu.cn

Yongfu Li received the Ph.D. degree in control science and engineering from Chongqing University, Chongqing, China, in 2012. He is currently a Professor of Control Science and Engineering with the Chongqing University of Posts and Telecommunications (CQUPT) and also the Director of the Key Laboratory of Intelligent Air-Ground Cooperative Control for Universities in Chongqing. His research interests include connected and automated vehicles, intelligent transportation systems, and UAV swarm control.

Taixiong Zheng received the Ph.D. degree in mechanical engineering from Chongqing University, Chongqing, China, in 2003. He is currently a Professor of control science and engineering with Chongqing University of Posts and Telecommunications, Chongqing, China. His research interests include engine control and vehicle active safe control.

Jiufei Luo received his $\mathrm{PhD}$ degree from Chongqing University, China, in 2015. He is currently an associate professor in Chongqing University of Posts and Telecommunications, China. His research interests include mechanical signal processing and intelligent vehicle control.

Zonghuan Guo received his $\mathrm{PhD}$ degree from Chongqing University, China, in 2016. He is currently a postdoctoral research fellow in Chongqing University, China. His research interests include mechanical signal processing and intelligent vehicle control. 


\title{
Emergency collision avoidance control strategy based on four-wheel steering and differential braking
}

\begin{abstract}
Path tracking control strategy of emergency collision avoidance is the research hotspot for intelligent vehicles, and active four-wheel steering and integrated chassis control such as differential braking are the development trend for the control system of intelligent vehicle. Considering both driving performance and path tracking performance, an active obstacle avoidance controller integrated with four-wheel steering (4WS), active rear steering (ARS) and differential braking control (RBC) based on adaptive model predictive theory (AMPC) is proposed. The designed active obstacle avoidance control architecture is composed of two parts, a supervisor and an MPC controller. The supervisor is responsible for selecting the appropriate control mode based on driving vehicle information and threshold of lateral and roll stability. In addition, a non-linear predict model is employed to obtain the future states of the driving vehicle. Then the AMPC is used to calculate the desired steering angle and differential braking toque when the driving stability indexes exceed the safety threshold. Finally, the proposed collision avoidance path tracking control strategy was simulated under emergency conditions via Carsim-Simulink co-simulation. The results show that the controller based on AMPC can be used to tracking the path of obstacle avoidance and has good performance in driving stability under emergencies.
\end{abstract}

Keywords: active obstacle avoidance, active steering, differential braking, adaptive MPC, intelligent vehicle

\section{Introduction}

Currently, active obstacle avoidance is becoming the standard associate system on most intelligent vehicles and it is regarded as the most effective way to reduce traffic crash accidents, including frontal crash, side crash, rear-end crash and so on [1-2]. The obstacle avoidance control mainly includes two ways of turning and braking according to the specific traffic scenes. For obstacle avoidance control, most researchers utilize a path planner and a tracking controller [3-4]. Claussmann, et al [5] presented a review of path planning techniques over the last decade for highway autonomous driving, and described their features, applications, challenges, and open issues in details for path planning. For 
path tracking in obstacle avoidance conditions, steering control is the most common and effective method. Thus, various types of steering control systems for intelligent vehicles have been proposed to enhance driving stability. The first proposal was four-wheel steering (4WS) system, mainly including active front steering (AFS) [6-7] and active rear steering (ARS) [8]. However, the active steering systems affect the path tracking performance greatly with respect to yaw rate tracking under emergency. And incorporating other actuators into the control system can improve the tracking and driving performance of a vehicle, especially in emergencies [9]. As the initial stage of the autonomous driving, advanced driving assistant systems can enhance driving safety by real-time obstacle warning and conditional intervention. But the ability is limited in emergency scenarios when an obstacle suddenly appears in the middle of the road [10]. The obstacle avoidance strategies mainly include smooth curve planner [11], fuzzy-based control [12], and optimal control methods [13]. However, the vehicle cannot safely track the planned path in emergency situations, and rollover also happens frequently in highspeed collision avoidance, which can cause fatal injuries accidents and has raised much concern, especially in the vehicle with high center of gravity $(\mathrm{CG})$, such as van, bus and SUV [14].

The vehicle loses stability usually due to the maneuvering in limit conditions by human factor aspects [15]. Although the active safety control technology has been commonly used in passenger cars [16-17]. For intelligent vehicles with high CG, rollover accidents maybe still occur. Thus, many approaches have been proposed to enhance the rollover stability, such as the active steering [18], active suspension [19], and differential rollover braking control (RBC) [20]. Emergency steering in highspeed obstacle avoidance is easy to cause rollover due to the generation of the large lateral acceleration [21]. Studies 
show differential braking and active steering are two useful strategies to restrain the rapid increasing of vehicle lateral acceleration, and prevent rollover indirectly [22].

Moreover, for path tracking and stability control in emergency situations, the rollover performance indicator is often ignored in tracking performance evaluation, which may cause the vehicle deviation from the target path under emergency. Thus, design of an integrated path tracking controller for highspeed obstacle avoidance is a hot problem [23]. Cui, Ding, Wu, and Zhou [24] designed an integrated collision avoidance system by active steering and active braking. To calculate the steering angle and improve the driving stability, a feedforward controller and a separate subsystem are used, simultaneously. However, it may not achieve the collision avoidance objectives when the control actions of the stability controller conflict with the path tracking controller. Qian, Wang, and Zhao [25] designed a front steering controller based on MPC and integrated with differential braking by SMC, which can ensure the accuracy of path tracking and achieve sufficient rollover stability. While parametric uncertainties, speed varying, bring a great challenge to the MPC controller.

The present work uses previous knowledge to improve the path tracking performance and lateral stability by front steering and braking control. We focus on vehicle path tracking and rollover prevention for emergency collision avoidance system used ARS and RBC. To this end, a composite control strategy composed of a supervisor and an MPC controller considering tracking performance, yaw stability characters, and anti-rollover performance is proposed. The executive AMPC module based on ARS and $\mathrm{RBC}$ is utilized to carry out the requirements of the supervisor module. Moreover, we are interested in both driving stability and tracking performance for vehicle active obstacle avoidance system, especially the roll stability under emergency, to do this, a priority weight will be given to the yaw and roll aspect of the AMPC even though the path tracking 
performance became worse. We compare our method versus the classic version of MPC with a uniform weight distribution, versus a AMPC version with the distribution skewed by the priority weight and versus a classic version of PID. We show that our method reduces the peak value of vehicle yaw rate, roll angle by $40 \%$ under sufficient tracking accuracy, which means the proposed controller can be used to tracking the path of obstacle avoidance and did well in driving stability. In addition, our results provide evidence that the method can prevent rollover under high-speed emergency.

Taking an SUV vehicle (SF5) as control object and the key parameters are shown in Table 1. The rest of this article is organized as follows. Section 2 describes the used vehicle dynamic models. The obstacle avoidance controller by 4WS and ARS based on AMPC theory is setup in Section 3. Then an integrated emergency collision avoidance controller is described in Section 4. Finally, conclusions are drawn in Section 5.

\section{Vehicle dynamics model}

\section{Two DOF vehicle model}

Figure 1 describes the vehicle dynamic characteristics of two DOF model used in this article.

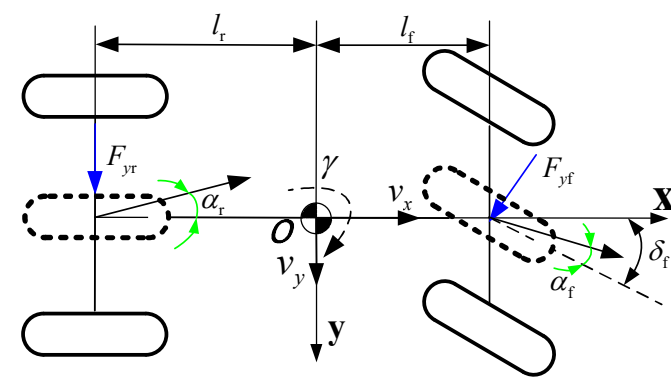

Figure 1. Two DOF vehicle dynamics model.

Assuming the vehicle runs under an ideal condition and the vehicle's lateral, yaw motion dynamic equations expressed, respectively, as

$$
m\left(\dot{v}_{y}+v_{x} \gamma\right)=F_{y \mathrm{f}} \cos \delta_{\mathrm{f}}+F_{y \mathrm{r}},
$$




$$
I_{z} \dot{\gamma}=l_{\mathrm{f}} F_{y \mathrm{f}} \cos \delta_{\mathrm{f}}-l_{\mathrm{r}} F_{\text {yr }}
$$

where, $m$ is the mass of vehicle, $v_{x}, v_{y}$ are the longitudinal and lateral velocity, $l_{\mathrm{f}}$ and $l_{\mathrm{r}}$ are the distances from $\mathrm{CG}$ to front and rear axle, respectively, $F_{y \mathrm{f}}$ and $F_{y \mathrm{r}}$ are vehicle's lateral forces, $\delta_{\mathrm{f}}$ is the steering angle of front wheel, $I_{\mathrm{z}}$ is the yaw moment of inertia.

The slip angle of the front and rear tire is obtained as

$$
\left\{\begin{array}{l}
\alpha_{\mathrm{f}}=\delta_{\mathrm{f}}-\frac{v_{y}+l_{\mathrm{f}} \gamma}{v_{x}} \\
\alpha_{\mathrm{r}}=\frac{-v_{y}+l_{\mathrm{r}} \gamma}{v_{x}}
\end{array}\right.
$$

Then, the linear tire model can be expressed as

$$
\begin{gathered}
F_{y f}=k_{\mathrm{f}} \alpha_{\mathrm{f}}, \\
F_{y \mathrm{r}}=k_{\mathrm{r}} \alpha_{\mathrm{r}},
\end{gathered}
$$

where $k_{\mathrm{f}}, k_{\mathrm{r}}$ are the vehicle cornering stiffness. Then, the vehicle's dynamic equations are obtained as

$$
\left\{\begin{array}{l}
m\left(\dot{v}_{y}+v_{x} \gamma\right)=k_{\mathrm{f}} \delta_{\mathrm{f}}-\frac{\left(k_{\mathrm{f}}+k_{\mathrm{r}}\right) v_{y}}{v_{x}}-\frac{\left(l_{\mathrm{f}} k_{\mathrm{f}}-l_{r} k_{\mathrm{r}}\right) \gamma}{v_{x}} \\
I_{z} \dot{\gamma}=l_{\mathrm{f}} k_{\mathrm{f}} \delta_{\mathrm{f}}-\frac{\left(l_{\mathrm{f}} k_{\mathrm{f}}-l_{\mathrm{r}} k_{\mathrm{r}}\right) v_{y}}{v_{x}}-\frac{\left(l_{\mathrm{f}}^{2} k_{\mathrm{f}}+l_{\mathrm{r}}^{2} k_{\mathrm{r}}\right) \gamma}{v_{x}}
\end{array} .\right.
$$

2-DOF linear model is regularly used by most scholars on active rear wheel steering design. The rear tire slip angle is obtained as

$$
\alpha_{\mathrm{r}}=\delta_{\mathrm{r}}-\frac{v_{y}-l_{\mathrm{r}} \gamma}{v_{x}}
$$

where $\delta_{\mathrm{r}}$ is the active rear wheels steering angle. Equation (6) can be expressed as

$$
\left\{\begin{array}{l}
m \dot{v}_{y}+\frac{\left(k_{\mathrm{f}}+k_{\mathrm{r}}\right) v_{y}}{v_{x}}+\left(m v_{x}+\frac{l_{\mathrm{f}} k_{\mathrm{f}}-l_{r} k_{\mathrm{r}}}{v_{x}}\right) \cdot \gamma=k_{\mathrm{f}} \delta_{\mathrm{f}}+k_{\mathrm{r}} \delta_{\mathrm{r}} \\
I_{z} \dot{\gamma}+\frac{\left(l_{\mathrm{f}}^{2} k_{\mathrm{f}}+l_{\mathrm{r}}^{2} k_{\mathrm{r}}\right) \gamma}{v_{x}}+\frac{\left(l_{\mathrm{f}} k_{\mathrm{f}}-l_{\mathrm{r}} k_{\mathrm{r}}\right) v_{y}}{v_{x}}=l_{\mathrm{f}} k_{\mathrm{f}} \delta_{\mathrm{f}}-l_{\mathrm{r}} k_{\mathrm{r}} \delta_{\mathrm{r}}
\end{array}\right.
$$

Written in state equation of the equation (8), as 
where, $\mathbf{x}=\left[v_{y}, \gamma\right], \mathbf{u}=\left[\delta_{\mathrm{f}}, \delta_{\mathrm{r}}\right]$. Also

$\mathbf{A}=-\frac{1}{v_{x}}\left[\begin{array}{cc}\frac{1}{m}\left(k_{\mathrm{f}}+k_{\mathrm{r}}\right) & \frac{1}{m}\left(l_{\mathrm{f}} k_{\mathrm{f}}-l_{r} k_{\mathrm{r}}\right)+v_{x}^{2} \\ \frac{l_{\mathrm{f}} k_{\mathrm{f}}-l_{\mathrm{r}} k_{\mathrm{r}}}{I_{z}} & \frac{l_{\mathrm{f}}^{2} k_{\mathrm{f}}+l_{\mathrm{r}}^{2} k_{\mathrm{r}}}{I_{z}}\end{array}\right], \mathbf{B}=\left[\begin{array}{cc}\frac{1}{m} k_{\mathrm{f}} & \frac{1}{m} k_{\mathrm{r}} \\ \frac{l_{\mathrm{f}} k_{\mathrm{f}}}{I_{z}} & -\frac{l_{\mathrm{r}} k_{\mathrm{r}}}{I_{z}}\end{array}\right]$.

Finding the characteristic roots of equation (9), can be written as

$$
\begin{gathered}
\operatorname{det}(s \mathbf{I}-\mathbf{A})=\left|\begin{array}{cc}
s+\frac{1}{m v_{x}}\left(k_{\mathrm{f}}+k_{\mathrm{r}}\right) & v_{x}+\frac{1}{m v_{x}}\left(l_{\mathrm{f}} k_{\mathrm{f}}-l_{\mathrm{r}} k_{\mathrm{r}}\right) \\
\frac{1}{I_{z} v_{x}}\left(l_{\mathrm{f}} k_{\mathrm{f}}-l_{\mathrm{r}} k_{\mathrm{r}}\right) & s+\frac{1}{I_{z} v_{x}}\left(l_{\mathrm{f}}^{2} k_{\mathrm{f}}+l_{\mathrm{r}}^{2} k_{\mathrm{r}}\right)
\end{array}\right|=0, \\
\Rightarrow \quad s^{2}+\left(\frac{l_{\mathrm{f}}^{2} k_{\mathrm{f}}+l_{\mathrm{r}}^{2} k_{\mathrm{r}}}{I_{z} v_{x}}+\frac{k_{\mathrm{f}}+k_{\mathrm{r}}}{m v_{x}}\right) s+\frac{k_{\mathrm{f}}+k_{\mathrm{r}}}{m v_{x}} \cdot \frac{l_{\mathrm{f}}^{2} k_{\mathrm{f}}+l_{\mathrm{r}}^{2} k_{\mathrm{r}}}{I_{z} v_{x}}, \\
\Rightarrow \quad-\frac{l_{\mathrm{f}} k_{\mathrm{f}}-l_{\mathrm{r}} k_{\mathrm{r}}}{I_{z} v_{x}} \cdot\left(\frac{l_{\mathrm{f}} k_{\mathrm{f}}-l_{r} k_{\mathrm{r}}}{m v_{x}}+v_{x}\right) A=0 \\
s^{2}+\left(\frac{l_{\mathrm{f}}^{2} k_{\mathrm{f}}+l_{\mathrm{r}}^{2} k_{\mathrm{r}}}{I_{z} v_{x}}+\frac{k_{\mathrm{f}}+k_{\mathrm{r}}}{m v_{x}}\right) s+\frac{l^{2} k_{\mathrm{f}} k_{\mathrm{r}}}{m I_{z} v_{x}^{2}}\left(1-\frac{m\left(l_{\mathrm{f}} k_{\mathrm{f}}-l_{r} k_{\mathrm{r}}\right)}{l^{2} k_{\mathrm{f}} k_{\mathrm{r}}} v_{x}^{2}\right)=0, \\
\Rightarrow s^{2}+\left(\frac{l_{\mathrm{f}}^{2} k_{\mathrm{f}}+l_{\mathrm{r}}^{2} k_{\mathrm{r}}}{I_{z} v_{x}}+\frac{k_{\mathrm{f}}+k_{\mathrm{r}}}{m v_{x}}\right) s+\frac{l^{2} k_{\mathrm{f}} k_{\mathrm{r}}}{m I_{z} v_{x}^{2}}\left(1+K v_{x}^{2}\right)=0,
\end{gathered}
$$

where, $K=\frac{m\left(l_{\mathrm{r}} k_{\mathrm{r}}-l_{\mathrm{f}} k_{\mathrm{f}}\right)}{l^{2} k_{\mathrm{f}} k_{\mathrm{r}}}$. It can be concluded from equation (13) that the 4WS vehicle is unstable if $K>-1 / v_{x}^{2}$.

In stationary situations, $\dot{v}_{y}=0, \dot{\gamma}=0$. Equation (8) can be expressed as

$$
\begin{gathered}
{\left[m v_{x}^{2}\left(l_{\mathrm{f}} k_{\mathrm{f}}-l_{\mathrm{r}} k_{\mathrm{r}}\right)-l^{2} k_{\mathrm{f}} k_{\mathrm{r}}\right] \gamma=-k_{\mathrm{f}} k_{\mathrm{r}} v_{x}\left(\delta_{\mathrm{f}}-\delta_{\mathrm{r}}\right),} \\
\Rightarrow\left(1+K v_{x}^{2}\right) \gamma=\frac{v_{x}}{l}\left(\delta_{\mathrm{f}}-\delta_{\mathrm{r}}\right) \\
\Rightarrow \delta_{\mathrm{f}}-\delta_{\mathrm{r}}=\frac{\gamma l}{v_{x}}\left(1+K v_{x}^{2}\right) .
\end{gathered}
$$


It has been proved that the lateral stability evaluation index of $\gamma$ is adjustable by control $\delta_{\mathrm{r}}$ before a vehicle reaching on limit operating condition (see Li, Zhao, Lin, \& Xiao, 2019). According to equation (16), the rear steering controller can be designed as

$$
\delta_{\mathrm{r}}=\delta_{\mathrm{f}}-\frac{\left(1+K v_{x}^{2}\right) l}{v_{x}} \cdot \gamma .
$$

\section{Front steering by preview-follower}

A ten-point preview steering control model is designed in Figure 2.

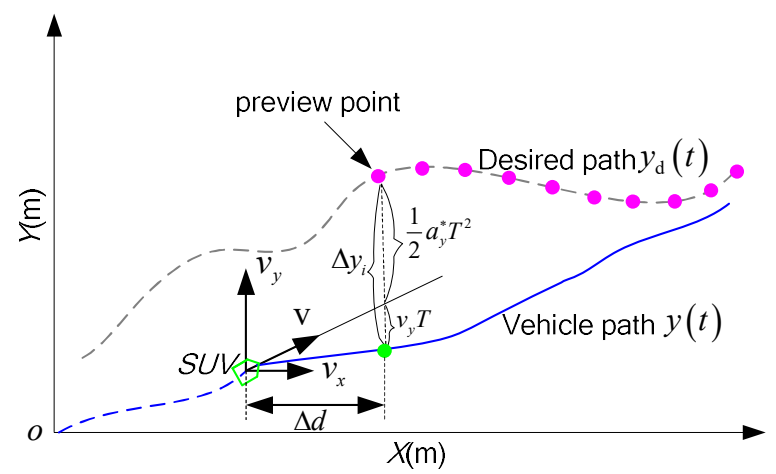

Figure 2. Preview-follower theory for steering control.

The preview distance is $\Delta d$. The error of lateral position between the desired path and the vehicle path can be defined as [23]

$$
\begin{gathered}
\Delta y_{i}=y_{\mathrm{d} i}(t+T)-y_{i}(t), \\
T=\Delta d / v_{x},
\end{gathered}
$$

where $y_{\mathrm{di}}(t), y_{i}(t)$ are the desired and actual lateral displacement, $T$ is the preview time, and $T=1 \mathrm{~s}$. It presumes that the tracking error $\Delta y_{i}$ can be eliminated after $T$. Thus,

$$
\begin{gathered}
y_{\mathrm{d}}(t)=y(t+T)+\Delta y_{i}^{*}, \\
\Rightarrow \Delta y_{i}^{*}=y(t+T)-y_{\mathrm{d}}(t)=\dot{y}(t) T+\frac{a_{y}^{*} T^{2}}{2},
\end{gathered}
$$

then

$$
a_{y}^{*}=\frac{2(y(t+T)-y(t)-\dot{y}(t) T)}{T^{2}},
$$


where, $a_{y}^{*}$ is the ideal value of $a_{y}$.

The realistic absolute value of $v_{y} \ll v_{x}$. Thus, the total velocity $\mathbf{v}=\sqrt{v_{x}^{2}+v_{y}^{2}} \approx v_{x}$. Since $\mathrm{v}=\gamma R$ (vehicle turning radius), then,

$$
a_{y}=\gamma^{2} R=\left(\frac{v_{x}}{R}\right)^{2} R=\gamma\left(\frac{v_{x}}{R}\right) R=\gamma v_{x},
$$

Substituted equation (23) for (17), then, the 4WS system is designed as

$$
\delta_{\mathrm{f}}=\delta_{\mathrm{r}}+\frac{\left(1+K v_{x}^{2}\right) l}{v_{x}^{2}} a_{y} \text {, }
$$

To achieve $a_{y}^{*}$ for front steering system $\left(\delta_{\mathrm{r}}=0\right), \delta_{\mathrm{f}}^{*}$ should be applied as

$$
\delta_{\mathrm{f}}^{*}=\frac{a_{y}^{*}}{G_{\mathrm{ay}}},
$$

where

$$
G_{\mathrm{ay}}=\frac{v_{x}^{2}}{l\left(1+K v_{x}^{2}\right)},
$$

The hierarchical architecture of the front steering by preview-follower is illustrated in Figure 3.

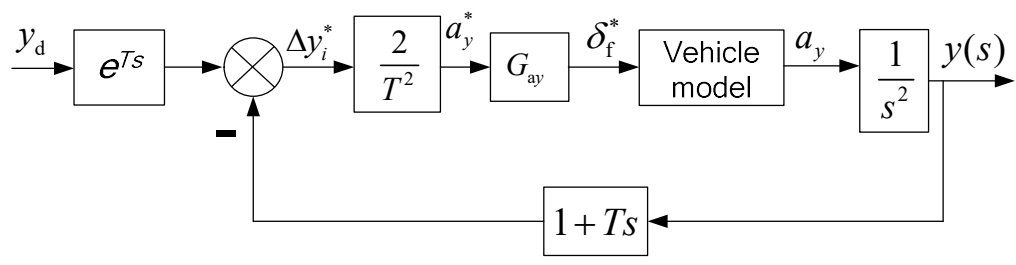

Figure 3. Block diagram of path tracking control scheme based on preview-follower.

\section{Carsim SUV vehicle model}

The Carsim simulation software is used for validation dynamics model and control strategy designed in this article. The four-wheel-steer controller can model in Matlab. Figure 4 
shows a SUV simulated in CarSim. A 275/65R18 radial pneumatic tire and generic front and rear independent suspensions are selected. The important parameters are given in Table 1.

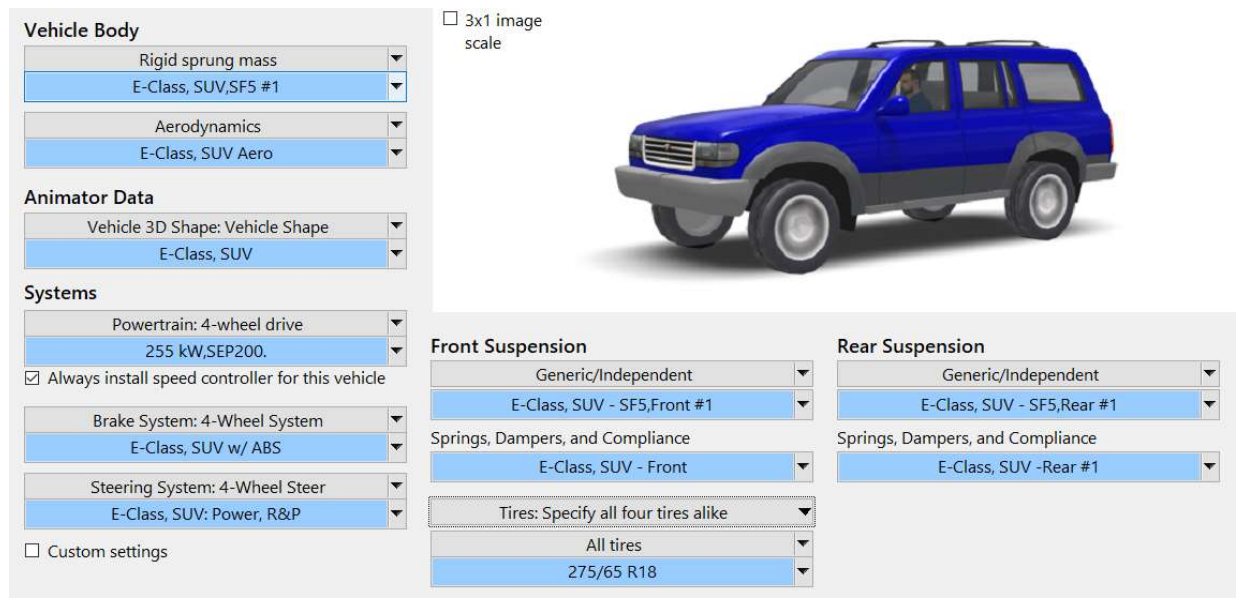

Figure 4. CarSim modeling interface.

Table 1. Important parameters for SUV.

\begin{tabular}{ll}
\hline Parameters & Value \\
\hline Sprung mass, total mass $m_{\mathrm{s}}, m$ & $2100,2370 \mathrm{~kg}$ \\
Front, rear unsprung mass $m_{\mathrm{uf}}, m_{\mathrm{ur}}$ & $120,150 \mathrm{~kg}$ \\
Front, rear axle distance to CG $l_{\mathrm{f}}, l_{\mathrm{r}}$ & $1.180,1.695 \mathrm{~m}$ \\
Wheel track width of front, rear axle $t_{\mathrm{wf}}, t_{\mathrm{wr}}$ & $1.655,1.650 \mathrm{~m}$ \\
Front, rear suspension roll stiffness $K_{\Phi \mathrm{f}}, K_{\Phi \mathrm{r}}$ & $92312,89311 \mathrm{Nm} / \mathrm{rad}$ \\
CG height to ground $h$ & $0.720 \mathrm{~m}$ \\
CG height to roll center $h_{\mathrm{s}}$ & $0.340 \mathrm{~m}$ \\
Wheel roll radius $r_{\mathrm{w}}$ & $0.390 \mathrm{~m}$ \\
Yaw moment of inertia $I_{z}$ & $2687 \mathrm{~kg} \cdot \mathrm{m}^{2}$ \\
Roll moment of inertia $I_{x}$ & $894.4 \mathrm{~kg} \cdot \mathrm{m}^{2}$ \\
Roll damping coefficient $C_{\Phi}$ & $5825 \mathrm{Nm} \cdot \mathrm{s} / \mathrm{rad}$ \\
Acceleration due to gravity $g$ & $9.81 \mathrm{~m} / \mathrm{s}^{2}$ \\
\hline
\end{tabular}

\section{Path tracking control strategy design}

Considering both handing performance and path tracking performance, an active obstacle avoidance path tracking controller integrated with 4WS, ARS based on AMPC theory is designed. The path tracking control structure is illustrated in Figure 5. 


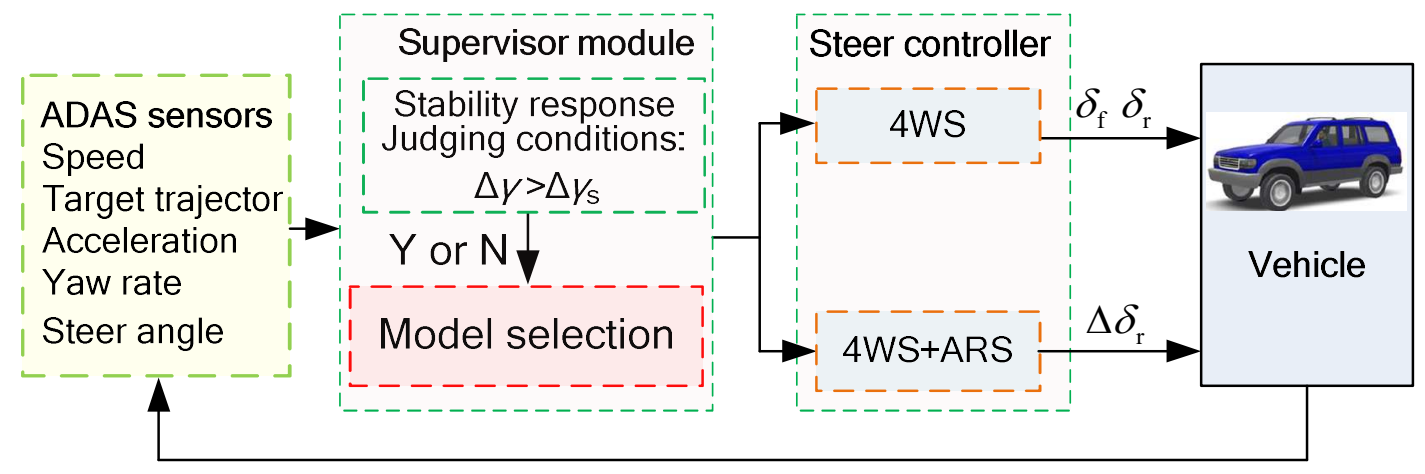

Figure 5. Path tracking control structure.

\section{General 4WS for obstacle avoidance system}

The hierarchical control architecture of the obstacle avoidance system by four-wheel steering is illustrated in Figure 6.

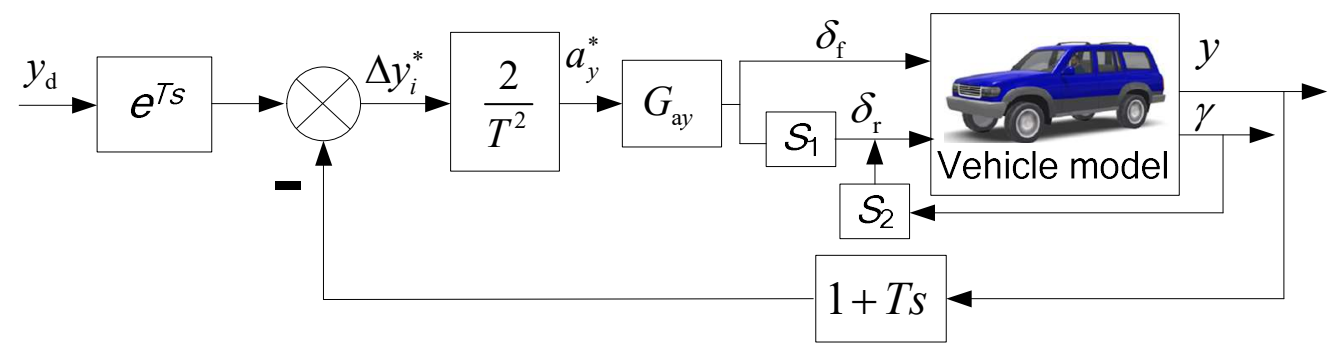

Figure 6. Hierarchical control architecture of 4WS.

To design the steering controller, the cornering stiffness of front and rear axle $k_{\text {f, }}$ $k_{\mathrm{r}}$ are set $110367 \mathrm{~N} / \mathrm{rad}, 70287 \mathrm{~N} / \mathrm{rad}$, respectively. According to equation (17), the gain coefficient of the four-wheel steering system $S_{1}=-1, S_{2}=-\frac{\left(1+K v_{x}^{2}\right) l}{v_{x}}$.

From the statistics of traffic accidents, changing lanes are very hazardous on busy highway. So, a double-lane-change (DLC) is utilized just like Figure 7, to verify the proposed 4WS system. The initial velocity is $120 \mathrm{~km} / \mathrm{h}$. The comparisons of the front and rear wheels steering angle, driving states response by 4WS and 2WS are shown in Figure 8. 


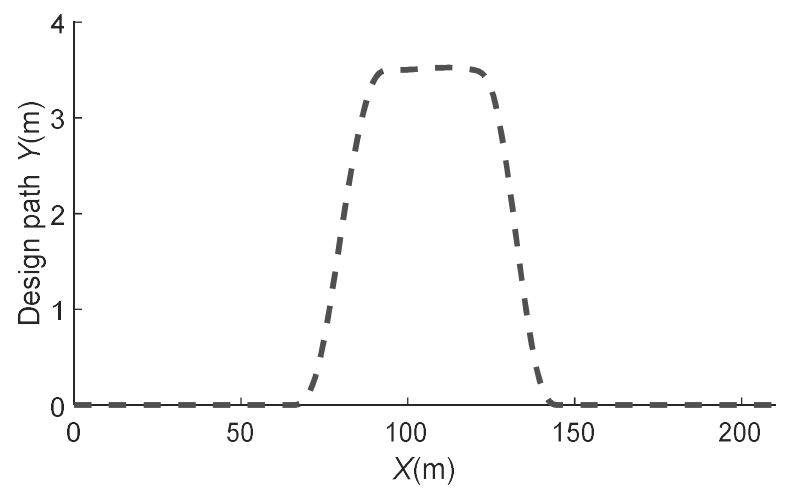

Figure 7. Design path of DLC (120 km/h).
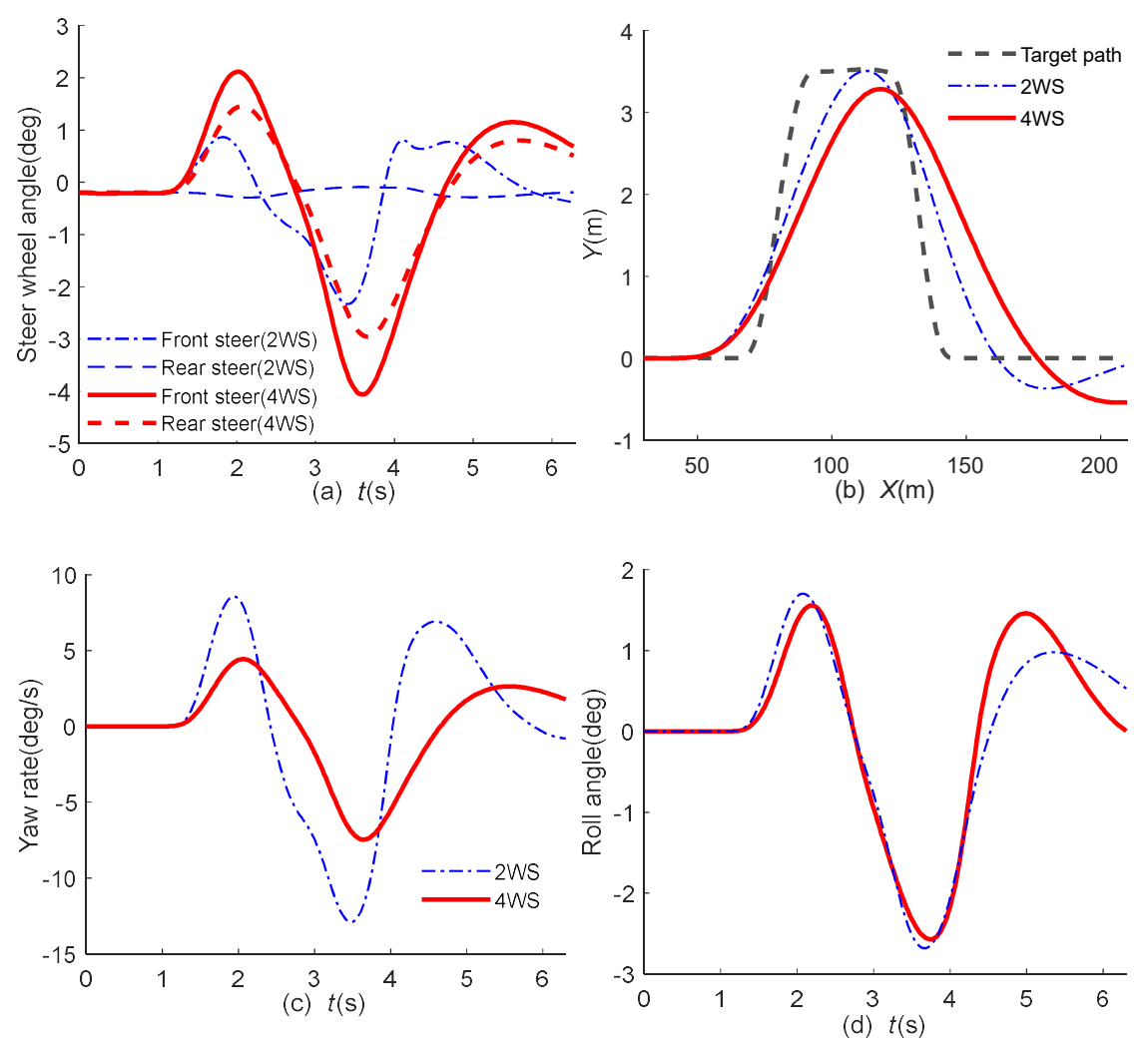

Figure 8. Simulation comparison with 4WS under the DLC condition: (a) Front, rear wheel steering angle; (b) lateral position; (c) Yaw rate; (d) Roll angle.

It can be observed from Figure 8 that the maximum of $\gamma$ has reduced $-46.04 \%$ at $3.5 \mathrm{~s}$ and the roll angle cut down $-30.1 \%$ at $5 \mathrm{~s}$ by $4 \mathrm{WS}$, respectively. However, the front steer angle increased $61.8 \%$ in the peak value at about $1.8 \mathrm{~s}$, and the path tracking performance was also reduced. Which means the general 4WS can help to improve the driving stability but will increase the front steer angle and path tracking error 
simultaneously. Figure 9 shows the 3D result of this scenario, where the blue and red cars are respectively related to $2 \mathrm{WS}$ and $4 \mathrm{WS}$.

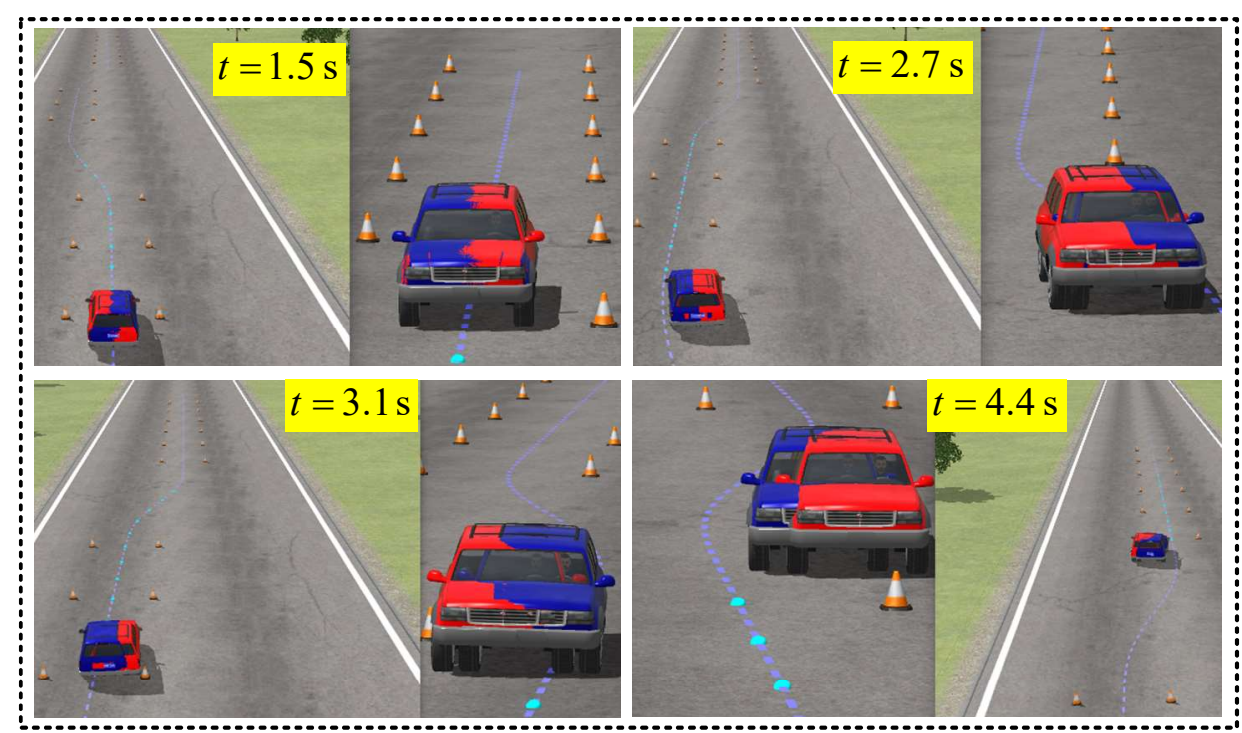

Figure 9. Dynamic visualization of obstacle avoidance path tracking (The blue and red cars are respectively related to $2 \mathrm{WS}$ and $4 \mathrm{WS}$ ).

\section{MPC Controller Design}

Through rolling optimization strategy, the MPC system can not only address the issues of tracking capability, parameters uncertain but also ensure driving stability. Therefore, the MPC based active steering is designed to track the design path, and the adaptive MPC system is introduced in this part.

Figure 10 shows the active rear wheel steer control flow charts by MPC.

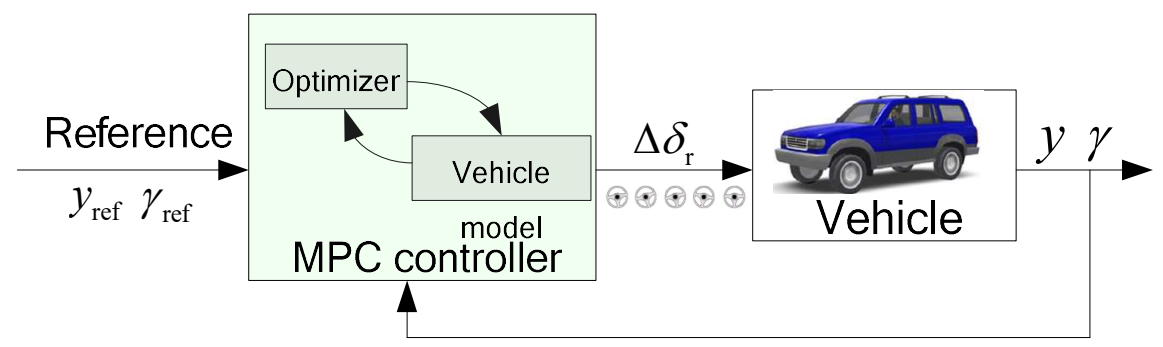

Figure 10. MPC controller of the active rear wheel steering.

For the linear vehicle model, the global $y$ position: 


$$
\dot{Y}=v_{x} \psi+v_{y},
$$

where $\psi$ is yaw angle.

The relevant equation of state is:

$$
\dot{x}=A x+B u,
$$

where, $x=\left[\begin{array}{lllll}y^{\prime} & \psi & \gamma & Y\end{array}\right], u=\left[\delta_{\mathrm{f}}, \delta_{\mathrm{r}}\right]$,

$$
A=\left[\begin{array}{cccc}
\frac{-1}{m v_{x}}\left(k_{\mathrm{f}}+k_{\mathrm{r}}\right) & 0 & -\frac{l_{\mathrm{f}} k_{\mathrm{f}}-l_{r} k_{\mathrm{r}}}{m v_{x}}-v_{x} & 0 \\
0 & 0 & 1 & 0 \\
-\frac{l_{\mathrm{f}} k_{\mathrm{f}}-l_{\mathrm{r}} k_{\mathrm{r}}}{I_{z} v_{x}} & 0 & -\frac{l_{\mathrm{f}}^{2} k_{\mathrm{f}}+l_{\mathrm{r}}^{2} k_{\mathrm{r}}}{I_{z} v_{x}} & 0 \\
1 & v_{x} & 0 & 0
\end{array}\right], \quad B=\left[\begin{array}{cccc}
\frac{k_{\mathrm{f}}}{m} & 0 & \frac{k_{\mathrm{r}}}{m} & 0 \\
\frac{l_{\mathrm{f}} k_{\mathrm{f}}}{I_{z}} & 0 & -\frac{l_{\mathrm{r}} k_{\mathrm{r}}}{I_{z}} & 0
\end{array}\right] .
$$

The discrete state space of equation (28) is achieved based on forward Euler method, as

$$
\begin{aligned}
& \dot{x}=\frac{1}{T}(x(k+1)-x(k))=A x(k)+B u(k), \\
& \Rightarrow x(k+1)=(T A+\mathrm{I}) x(k)+T B u(k), \\
& \Rightarrow x(k+1)=\tilde{A} \cdot x(k)+\tilde{B} \cdot u(k),
\end{aligned}
$$

where, $\tilde{A}=T A+\mathrm{I}, \tilde{B}=T B, x(k)$ are the vehicle states at $k$ time, $x(k+1)$ are the vehicle states at $k+1$ time, $\mathbf{I}$ is a unit matrix, $T$ is the discretization time.

A unique feature of the MPC method is that it can forecast the system's future state. The predicted state within $P$ control cycle as:

$$
\begin{gathered}
X_{k}=\left[x(k+1 \mid k)^{T}, x(k+2 \mid k)^{T}, \cdots, x\left(k+N_{p} \mid k\right)^{T}\right]^{T}, \\
U_{k}=\left[u(k \mid k)^{T}, u(k+1 \mid k)^{T}, \cdots, u\left(k+N_{c}-1 \mid k\right)^{T}\right]^{T},
\end{gathered}
$$


where, $x(k+1 \mid k), u(k+1 \mid k)$ are the states predicted at $k+1$ time, computed at $k$ time, respectively, $N_{p}$ is the predictive step length, $N_{c}$ is the control step length.

The system states of the future $P$ control periods are predicted by discretization of the state equations as:

$$
\begin{gathered}
x(k+1 \mid k)=\tilde{A} \cdot x(k)+\tilde{B} \cdot u(k \mid k), \\
x(k+2 \mid k)=\tilde{A}^{2} \cdot x(k)+\tilde{A} \tilde{B} \cdot u(k \mid k)+\tilde{B} \cdot u(k+1 \mid k), \\
x(k+3 \mid k)=\tilde{A}^{3} \cdot x(k)+\tilde{A}^{2} \tilde{B} \cdot u(k \mid k)+\tilde{A} \tilde{B} \cdot u(k+1 \mid k)+\tilde{B} \cdot u(k+2 \mid k), \\
x(k+P \mid k)=\tilde{A}^{P} \cdot x(k)+\sum_{i=0}^{P-1} \tilde{A}^{P-1-i} \tilde{B} \cdot u(k+i \mid k) .
\end{gathered}
$$

Written in state matrix form, then

$$
X_{k}=\phi x(k)+\varphi U_{k},
$$

where, $\phi=\left[\tilde{A}, \tilde{A}^{2} \cdots \tilde{A}^{P}\right]^{T}, \varphi=\left[\begin{array}{cccc}\tilde{A}^{1-1} \tilde{B} & \ldots & 0 & 0 \\ \tilde{A}^{2-1} \tilde{B} & \tilde{A}^{2-2} \tilde{B} & \cdots & 0 \\ \vdots & \vdots & \ddots & \vdots \\ \tilde{A}^{P-1} \tilde{B} & \tilde{A}^{P-2} \tilde{B} & \cdots & \tilde{A}^{P-P} \tilde{B}\end{array}\right]$.

Define a sequence of reference values in the predicted $P$ time as:

$$
R_{k}=\left[r_{r e f}(k+1)^{T}, r_{r e f}(k+2)^{T}, \cdots, r_{r e f}(k+P)^{T}\right]^{T} .
$$

where, $r_{\text {ref }}=\left[Y_{\text {ref,local }}, \gamma_{\text {ref, local }}\right]$.

According to the cumulative error between the predicted state vector and the reference value, the optimization objective function considering the constraints is as follows:

$$
J\left(U_{k}\right)=\sum_{i=1}^{N_{p}}\left\|X_{k}-R_{k}\right\|_{Q}^{2}+\sum_{i=0}^{N_{c-1}}\left|U_{k}\right|_{R}^{2}
$$

where $Q$ and $R$ are the weight matrixes. 
The MPC is an optimal control method. Combining Equations (38-40), the optimization problems can be solved for the active rear steering controller as

$$
\min _{\Delta u, \varepsilon}\{J(x(t), u(t-1), \Delta u(t))\},
$$

$$
\text { Subject to: } x(k+1)=\tilde{A} \cdot x(k)+\tilde{B} \cdot u(k) \text {, }
$$

$$
\begin{gathered}
u_{\text {min }}(k) \leq u_{t}(k) \leq u_{\text {max }}(k), \\
\Delta u_{\text {min }}(k) \leq \Delta u_{t}(k) \leq \Delta u_{\text {max }}(k), \\
a_{y, \text { min }}-\varepsilon \leq a_{y} \leq a_{y, \text { min }}+\varepsilon, \\
\varepsilon>0,
\end{gathered}
$$

where, $\left|a_{y}\right| \leq u g$.

The reference path is generated by a DLC for tracking controller. The MPC simulation parameters setting as: $N_{p}=20, N_{c}=2, \mathrm{r}=1000, Q=[2,0.2] . R=[0,1], \varepsilon=1$. The path tracking results, yaw stability index, rear steering angle comparisons by MPC $+4 \mathrm{WS}$ are shown in Figure 11.
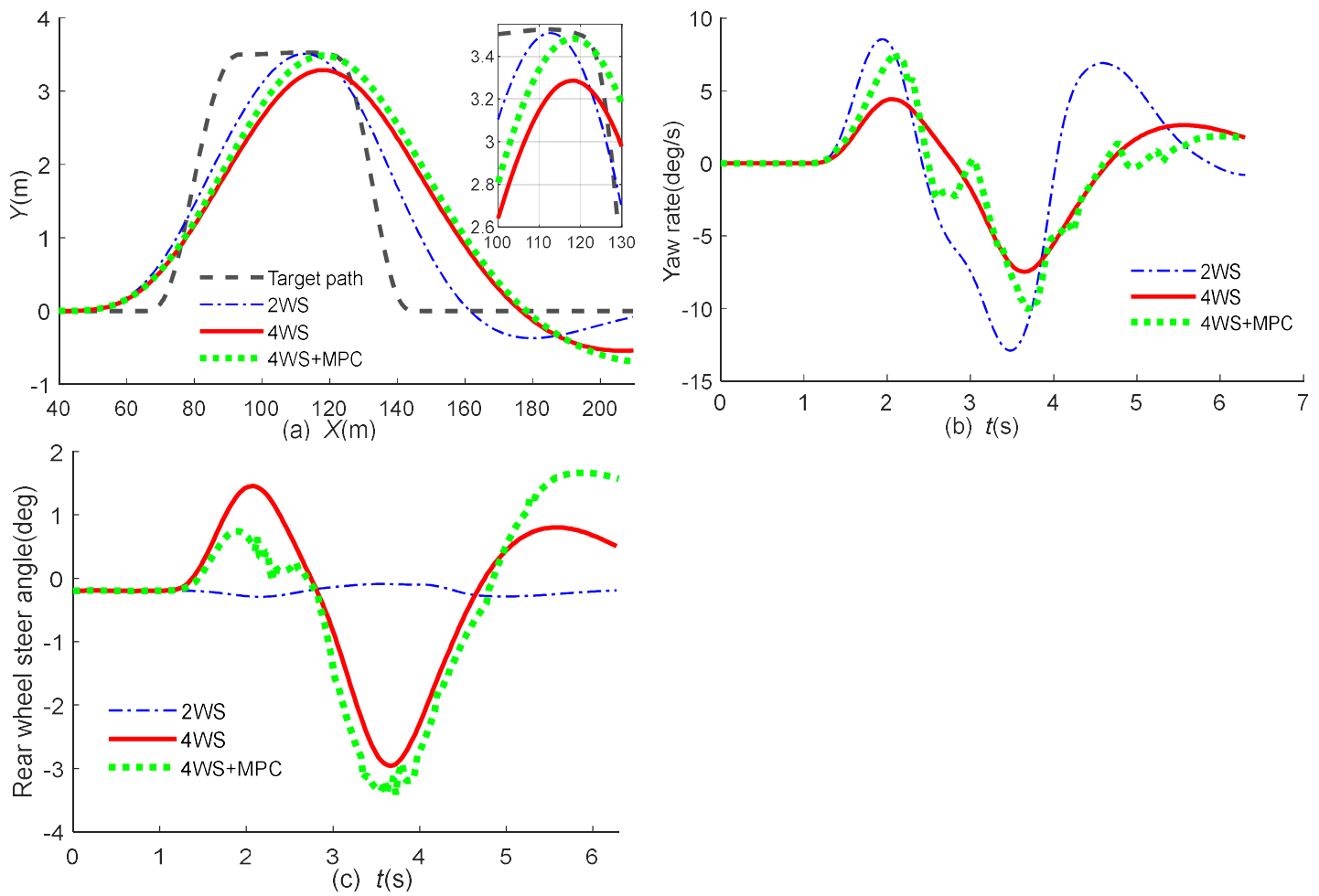

Figure 11. State response comparison by different controller: (a) Path tracking; (b) Yaw rate; (c) Rear steering angle. 
It can be seen from Figures 11 that the peak value of vehicle yaw rate cut down $30 \%$ by $4 \mathrm{WS}+\mathrm{MPC}$ at $3.5 \mathrm{~s}$ compared with $2 \mathrm{WS}$ and path tracking performance was also enhanced compared with 4WS. In addition, the MPC has a negative effect on rear wheel steering at $2 \mathrm{~s}$ for good accuracy of path tracking. In other words, the $4 \mathrm{WS}$ with MPC controller can help to improve the driving stability and tracking performance simultaneously. The results given in Figure 12 show the tracking performance and stability indexes of 4WS+MPC in different weight matrixes of $Q\left(\left[\begin{array}{ll}0.8 & 0.2\end{array}\right],\left[\begin{array}{ll}0.4 & 0.6\end{array}\right],[0.2\right.$ $0.8])$, respectively.
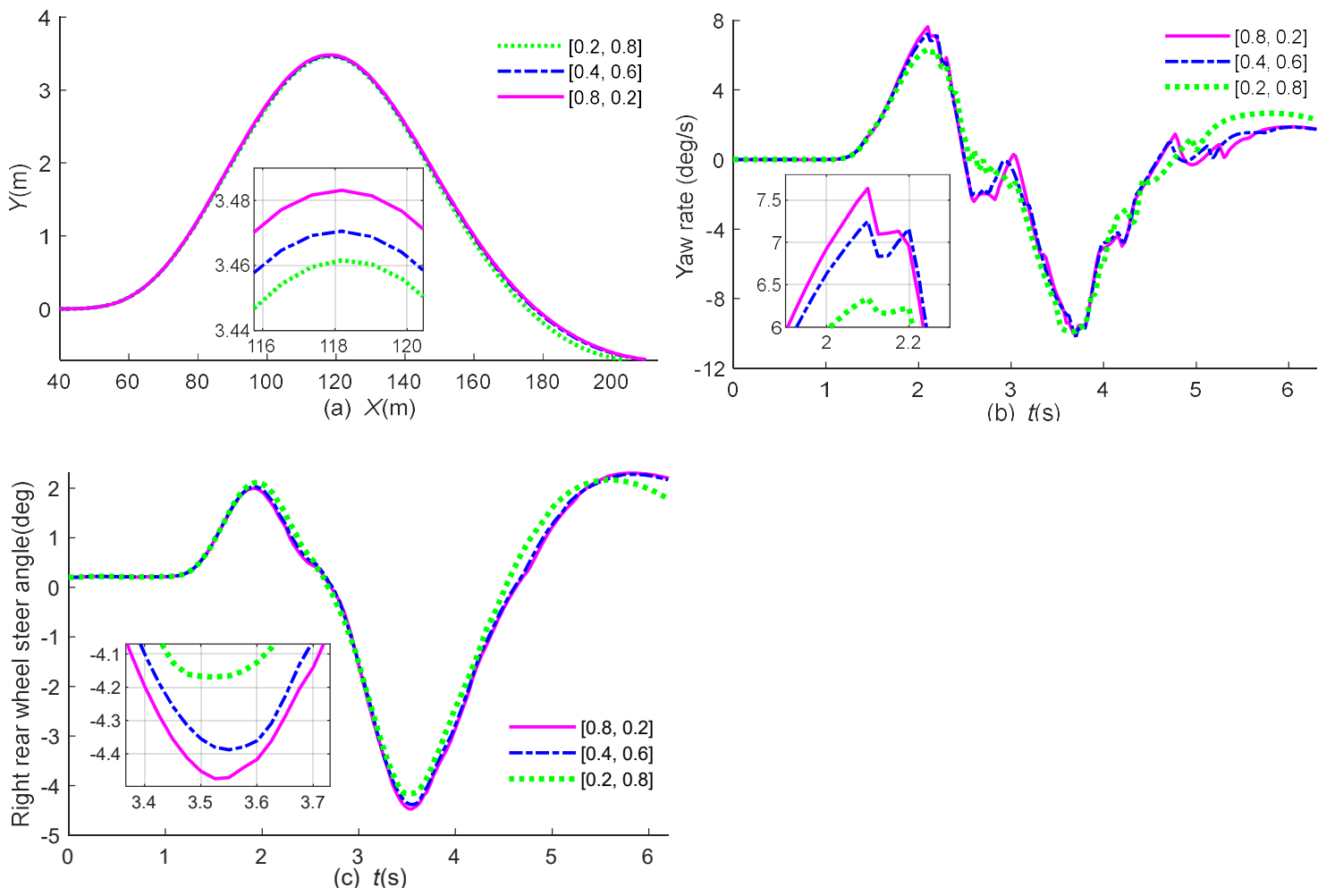

Figure 12. State response comparison at different weight matrixes: (a) Path tracking; (b) Yaw rate; (c) Rear steering angle.

It can be found from Figure $12(b, c)$ that the weight of path tracking index increases with the error between yaw rate and its reference values. For $4 \mathrm{WS}+\mathrm{MPC}$ with high weight of lateral displacement, the steering angle of rear wheel and yaw rate response should be larger than $4 \mathrm{WS}+\mathrm{MPC}$ with low weight. 4WS+MPC with high weight 
of yaw rate index can give better stability control performance because it can get more weight considerations in the process of optimization control.

Figure 13 shows the vehicle tracking accuracy and stability indexes of $4 \mathrm{WS}+\mathrm{MPC}$ in various velocity $(80 \mathrm{~km} / \mathrm{h}, 100 \mathrm{~km} / \mathrm{h}, 120 \mathrm{~km} / \mathrm{h})$, respectively.
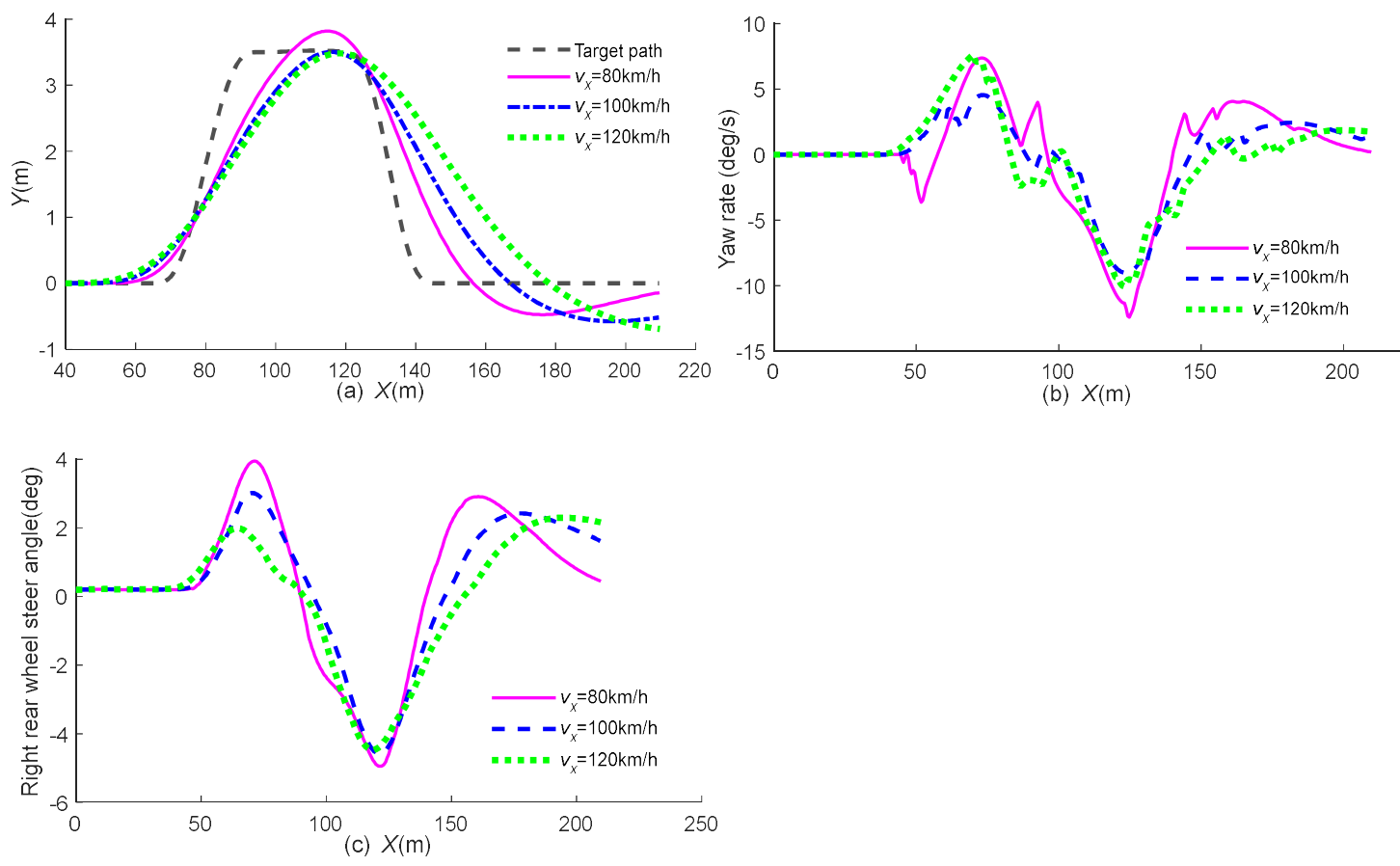

Figure 13. State response comparison at different longitudinal speed: (a) Path tracking; (b) Yaw rate; (c) Rear steering angle.

In Figure 13, it concluded that the peak value of lateral station, yaw rate and rear wheel steering angle changed obviously, when $v_{\mathrm{x}}=80 \mathrm{~km} / \mathrm{h}$, tracking accuracy is good, but the stability index of yaw gets worse. When $v_{\mathrm{x}}=120 \mathrm{~km} / \mathrm{h}$, vehicle driving stability is best, but tracking accuracy gets worse. In other words, it means the proposed path tracking control strategy based on MPC cannot adapt to vehicle speed change completely.

\section{Adaptive MPC Controller Design}

In the MPC controller, it assumed that the vehicle driving at a constant speed. The vehicle dynamics don't change and A (state matrix) is constant. But if the longitudinal speed varies as the vehicle travels, A also changes, and the conventional MPC cannot 
hand the nonlinear dynamics because it employs a fixed interior vehicle model. Thus, the adaptive MPC Controller (A combination of MPC and on-line update of the model parameters) is designed to deal with the changing vehicle dynamics. Figure 14 showed the structure of the adaptive MPC.

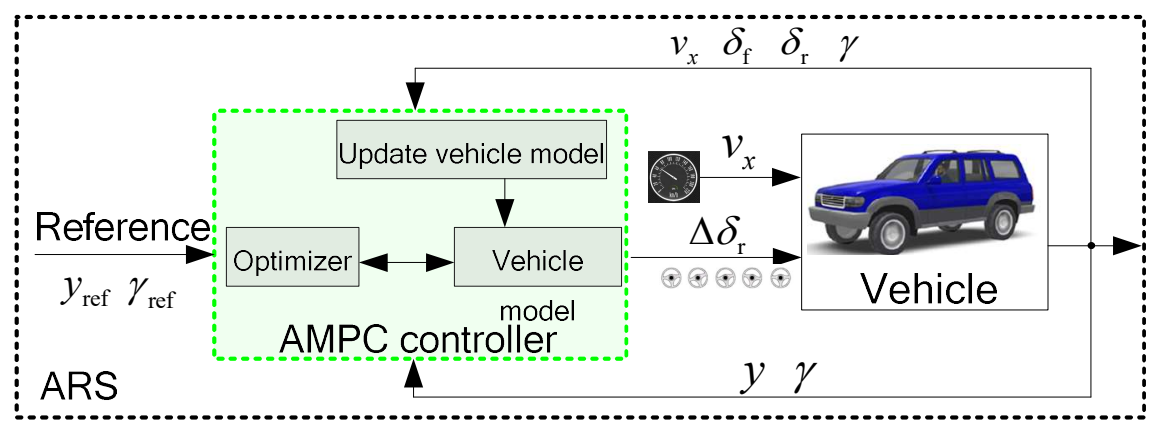

Figure 14. The structure of the adaptive MPC controller.

The pre-built function of the adaptive MPC takes $v_{x}$, steering angle and vehicle state as inputs. To make the vehicle path tracking controller fit for emergency collision avoidance, the preferred weights will be applied to the stability aspect and active rear wheel based on AMPC will be used even though the path tracking performance became worse. The "high-speed" denotes the dynamic velocity greater than $80 \mathrm{~km} / \mathrm{h}$ in this article. The tracking accuracy and stability indexes by different controllers are displayed in Figure 15.
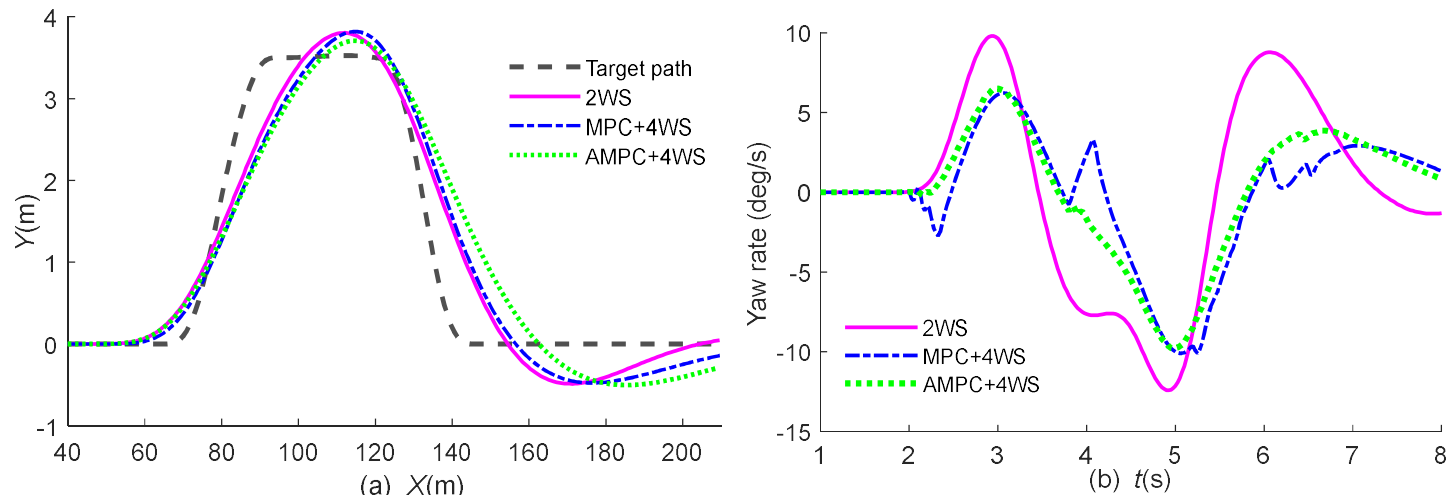


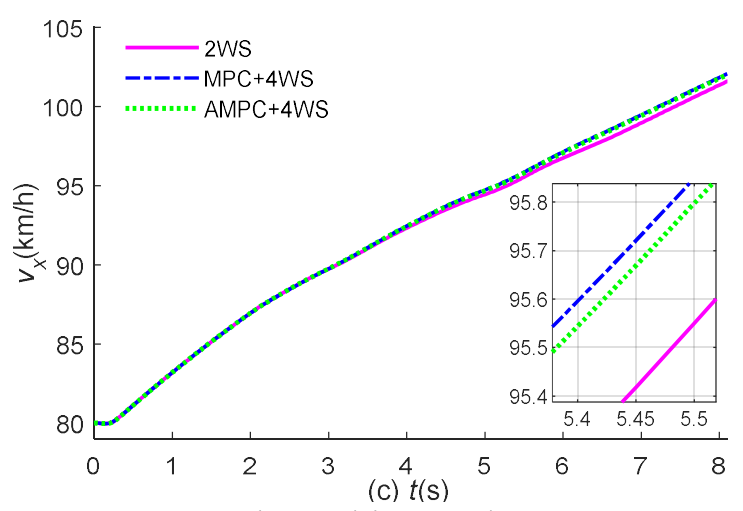

Figure 15. Path tracking and state response comparison by different controller: (a) Path tracking; (b) Yaw rate; (c) Vehicle longitudinal speed.

In Figure 15, vehicle yaw rate reduced more than $30 \%$ in peak value by AMPC $+4 \mathrm{WS}$ compared with general vehicle (2WS), and the AMPC $+4 \mathrm{WS}$ is better adapt to variable speed condition under tracking obstacle avoidance compared with MPC+4WS controller.

\section{Integrated active obstacle avoidance control design}

For SUV vehicle, rollover maybe still occur even with 4WS+ARS system on account of the high CG. In this section, an improved active collision avoidance controller integrated with 4WS, ARS and RBC based on AMPC theory is designed. Figure 16 shows the control flow charts of the integrated controller. The supervisory module decides the corresponding mode based on the ADAS sensors signal and safety thresholds. The underlying controller is based on $4 \mathrm{WS}, \mathrm{ARS}$, and RBC to carry out the supervisory module's requirements.

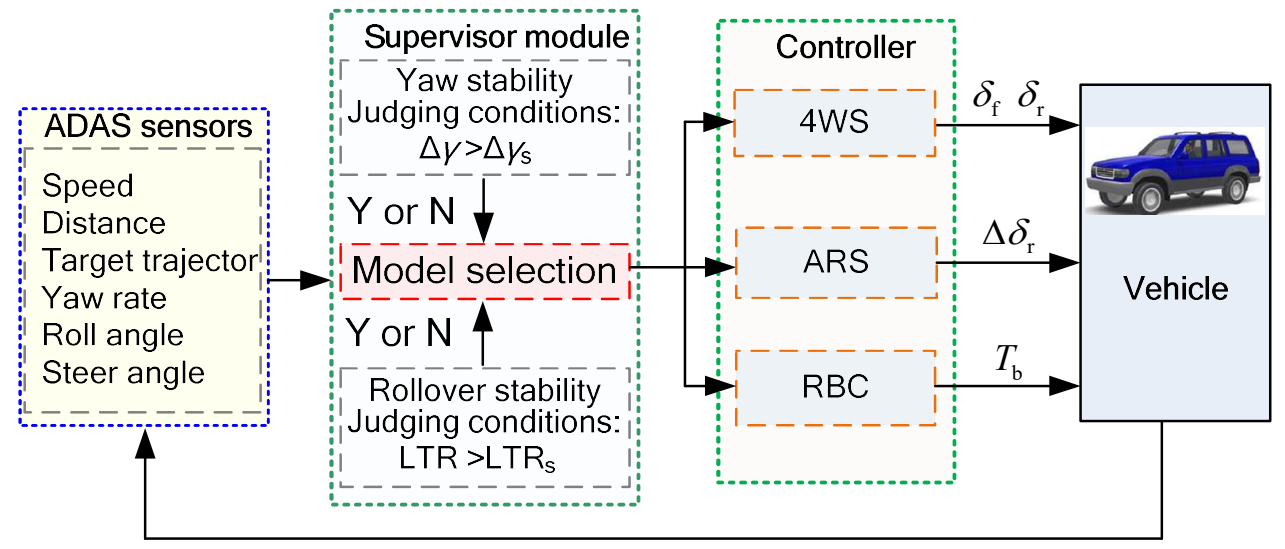

Figure 16. Structure of the integrated active obstacle avoidance system. 


\section{Rollover braking control strategy}

The hierarchical control architecture of the AMPC rollover controller is illustrated in Figure 17.

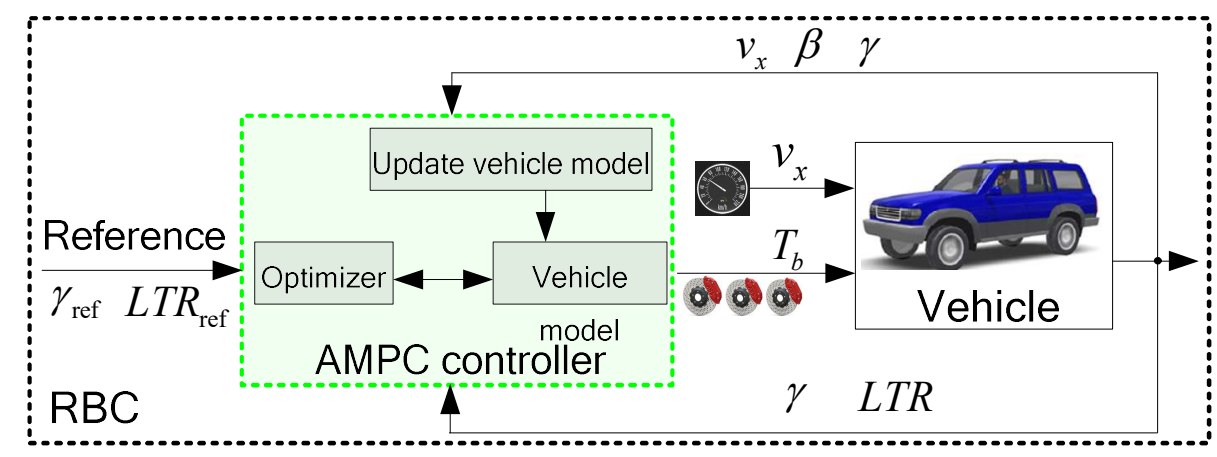

Figure 17. Hierarchical control architecture of the AMPC.

For the rollover control, design a rollover prediction module is needed. Load transfer ratio (LTR) is commonly used as

$$
L T R=\frac{F_{z \mathrm{r}}-F_{z 1}}{F_{z \mathrm{r}}+F_{z 1}}
$$

where, $F_{z \mathrm{r}}, F_{z 1}$ are right, left vertical loads on wheel.

By analyzing the vehicle mechanism of roll, the LTR rewrite as (see Li, Lu, Wang, \& Chen, 2017):

$$
L T R=\frac{2 C_{\phi} p+2\left(K_{\phi \mathrm{f}}+K_{\phi \mathrm{r}}\right) \phi}{m g t_{\mathrm{w}}} .
$$

If $|\mathrm{LTR}|$ is larger than 0.8 , it means vehicles in danger of rollover greatly. So, the threshold value of $\mathrm{LTR}_{\mathrm{S}}=0.8$. When rollover is about to occur, the braking instruction is ordered for rollover control, however, the brake force may too large and to prevent wheels from locking, the ABS controller [26] is also added.

To get the braking force as $|\mathrm{LTR}|>\mathrm{LTR}_{\mathrm{S}}$, an adaptive MPC is used to calculated the braking torque. Taking the 4-DOF vehicle model as the bases for rollover controller which is given in Figure 18. 


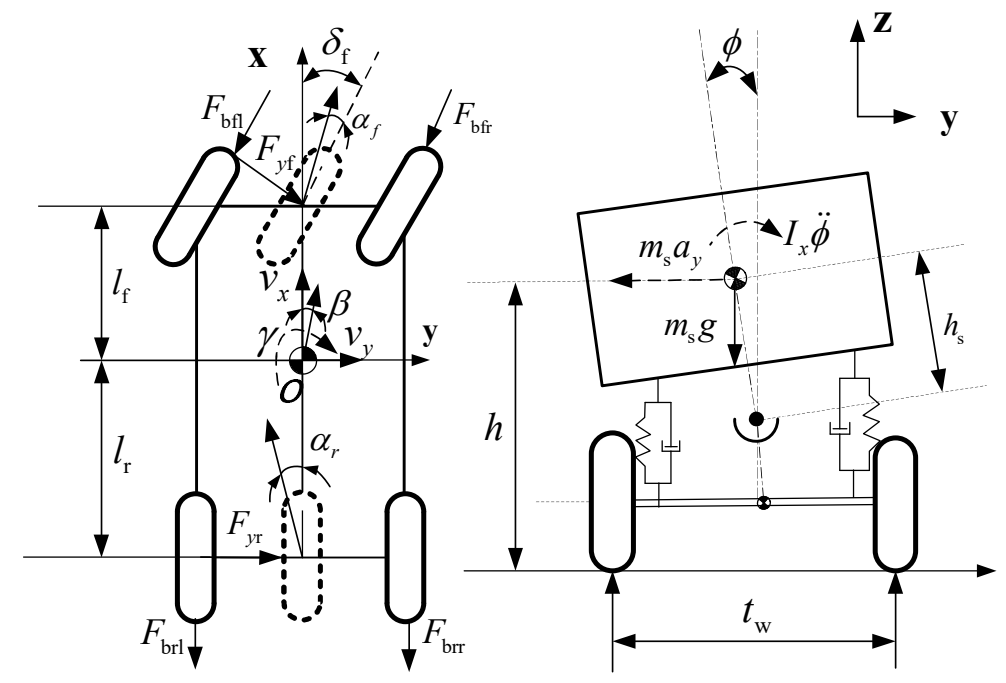

Figure 18. 4-DOF linear vehicle mode.

The equations of body motion for 4-DOF model can be described, respectively,

$$
\begin{gathered}
m\left(\dot{v}_{x}-\beta v_{x} \gamma\right)=\left(F_{\mathrm{bfl}}+F_{\mathrm{bfr}}\right) \cos \delta_{f}+F_{\mathrm{brl}}+F_{\mathrm{brr}}, \\
m v_{x}(\gamma+\dot{\beta})=F_{y \mathrm{f}} \cos \delta_{f}+F_{\mathrm{yr}}, \\
I_{z} \dot{\gamma}=l_{\mathrm{f}} F_{y \mathrm{f}}-l_{\mathrm{r}} F_{y \mathrm{r}}-\left(F_{\mathrm{bfl}}+F_{\mathrm{brl}}-F_{\mathrm{bfr}}-F_{\mathrm{brr}}\right) t_{\mathrm{w}} / 2, \\
I_{x} \dot{p}=m_{\mathrm{s}} a_{y} h_{\mathrm{s}}-C_{\phi} p+m_{s} g h_{s} \phi-\left(K_{\phi \mathrm{f}}+K_{\phi \mathrm{r}}\right) \phi,
\end{gathered}
$$

Considering Eqs. (45-48), the dynamic equations of vehicle can be rewritten as

$$
\dot{x}=f(x)+N u,
$$

where, the state variables $x=\left[v_{x} \gamma \beta \phi p\right]^{\mathrm{T}}$, the inputs of the AMPC are tire forces of the 4 wheels, and $u=\left[F_{\mathrm{b} x \mathrm{fl}} F_{\mathrm{b} x \mathrm{fr}} F_{\mathrm{bxrl}} F_{\mathrm{b} x \mathrm{rr}}\right]$,

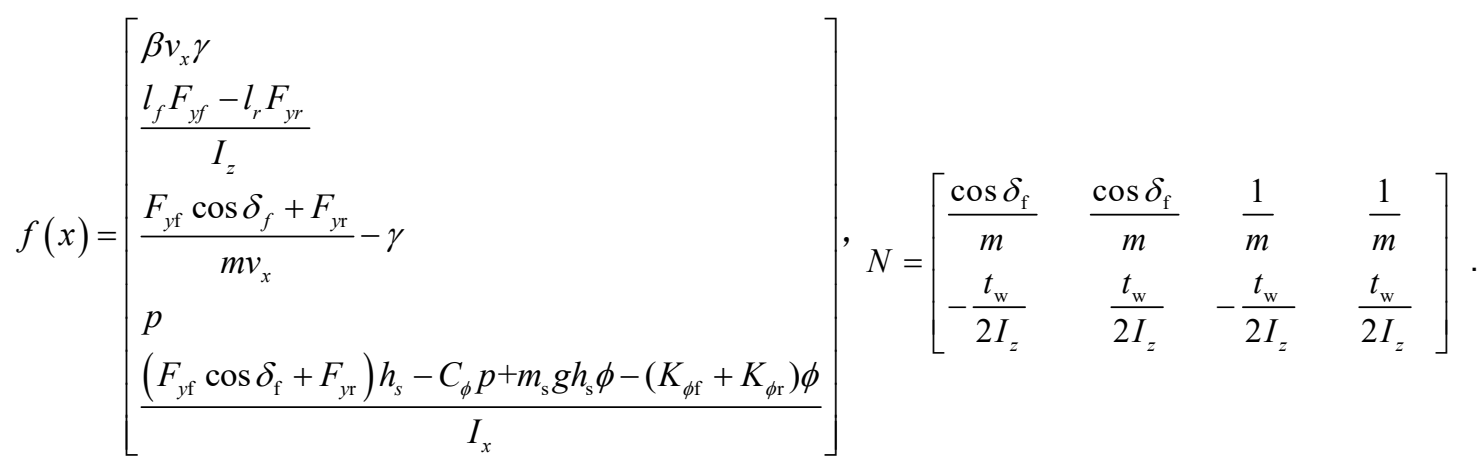

Then, its discrete and incremental form is represented as: 


$$
\Delta x(k+1)=A^{\prime} \cdot \Delta x(k)+B^{\prime} \cdot \Delta u(k),
$$

where, $A^{\prime}=\mathrm{I}+\left.T \cdot \frac{\partial f}{\partial x}\right|_{x(k)}, B^{\prime}=T N$.

The controlled output $X^{\prime}{ }_{k}$ is defined as yaw rate $\gamma$ and LTR.

$$
X^{\prime}=\left[\begin{array}{ll}
\gamma & \mathrm{LTR}
\end{array}\right]^{\mathrm{T}}=C^{\prime} x,
$$

where, $C^{\prime}=\left[\begin{array}{ccccc}0 & 1 & 0 & 0 & 0 \\ 0 & 0 & 0 & \frac{2\left(K_{\phi \mathrm{f}}+K_{\phi \mathrm{r}}\right)}{m g t_{w}} & \frac{2 C_{\phi}}{m g t_{w}}\end{array}\right]$.

Considering the actuator's ability, the input of the AMPC controller should satisfy

$$
u_{\min }^{\prime}(k) \leq u_{t}^{\prime}(k) \leq u_{\max }^{\prime}(k),
$$

The output needs to follow the references and minimize the input simultaneously. Thus, the AMPC cost function is designed as

$$
J^{\prime}\left(U_{k}\right)=\sum_{i=1}^{N_{p}}\left\|X_{k}^{\prime}-R_{k}^{\prime}\right\|_{Q}^{2}+\sum_{i=0}^{N_{c-1}}\left|U_{k}^{\prime}\right|_{R}^{2} .
$$

The optimization problems can be solved for the active controller as

$$
\min _{\Delta u^{\prime}, \varepsilon}\left\{J\left(x(t), u(t-1), \Delta u(t), N_{p}, N_{c}\right)\right\},
$$

Subject to: $x(k+1)=\tilde{A}^{\prime} x(k)+\tilde{B}^{\prime} u(k)$,

$$
\begin{gathered}
u_{\min }^{\prime}(k) \leq u_{t}^{\prime}(k) \leq u_{\max }^{\prime}(k), \\
\Delta u_{\min }^{\prime}(k) \leq \Delta u_{t}^{\prime}(k) \leq \Delta u_{\max }^{\prime}(k), \\
a_{x, \text { min }}-\varepsilon \leq a_{x} \leq a_{x, \text { min }}+\varepsilon, \\
\varepsilon>0,
\end{gathered}
$$

where, $\left|a_{x}\right| \leq u g$. 
To verify the effectiveness of the LTR by equation (44), a same "Sine" steering input is conducted to the vehicle at different speed, and Figure 19 shows the transient response of LTR at different initial speed.

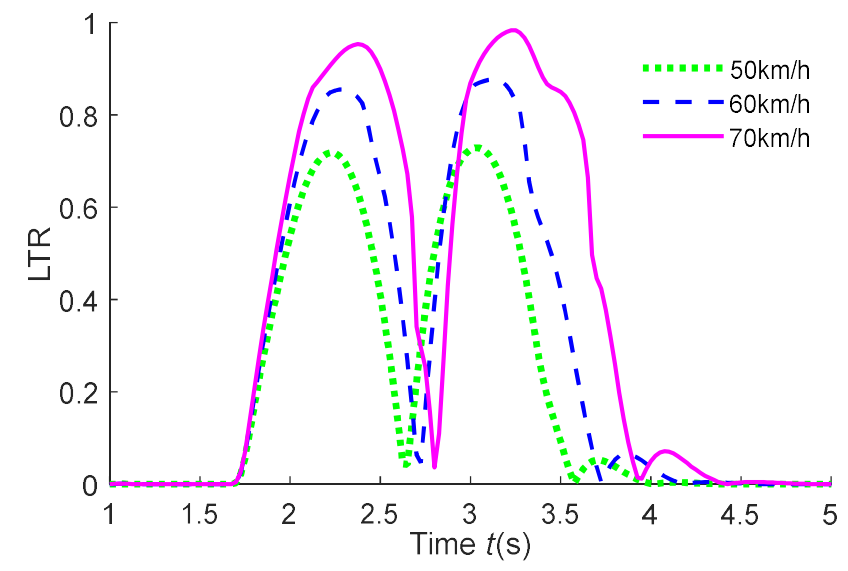

Figure 19. Transient response of LTR at different initial speed.

Figure 19 indicates that the rollover risk increases with increasing the vehicle speed, especially vehicle speed over $70 \mathrm{~km} / \mathrm{h}$. And the vehicle rollover index reaches its maximum threshold limit (LTR $\approx 1$ ) when avoiding the obstacles with the same "Sine" steering input. Rollover status approaching at $t=3.3 \mathrm{~s}$ and LTR can estimate the point.

A traffic accident accrued ahead of the vehicle in highway is adopted to verify the rollover control based on AMPC, and suppose that the 4WS+ARS is not working. The vehicle needs to avoid the obstacles immediately. The dynamic visualization is displayed in Figure 20, and $v_{x}=110 \mathrm{~km} / \mathrm{h}$.

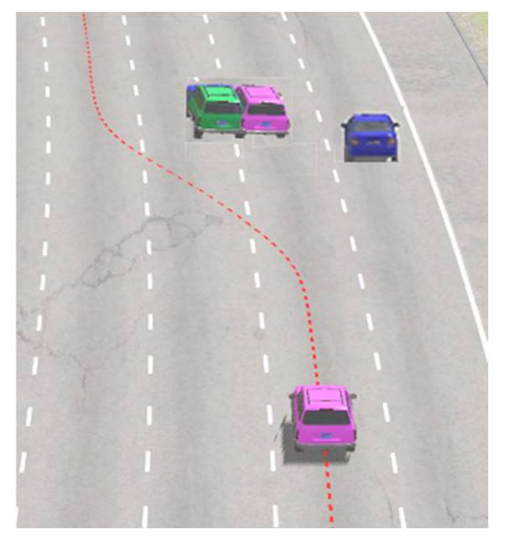

Figure 20. Dynamic visualization in highway emergency collision avoidance. 
Figure 21 shows the stability indexes comparisons by AMPC and traditional PID.
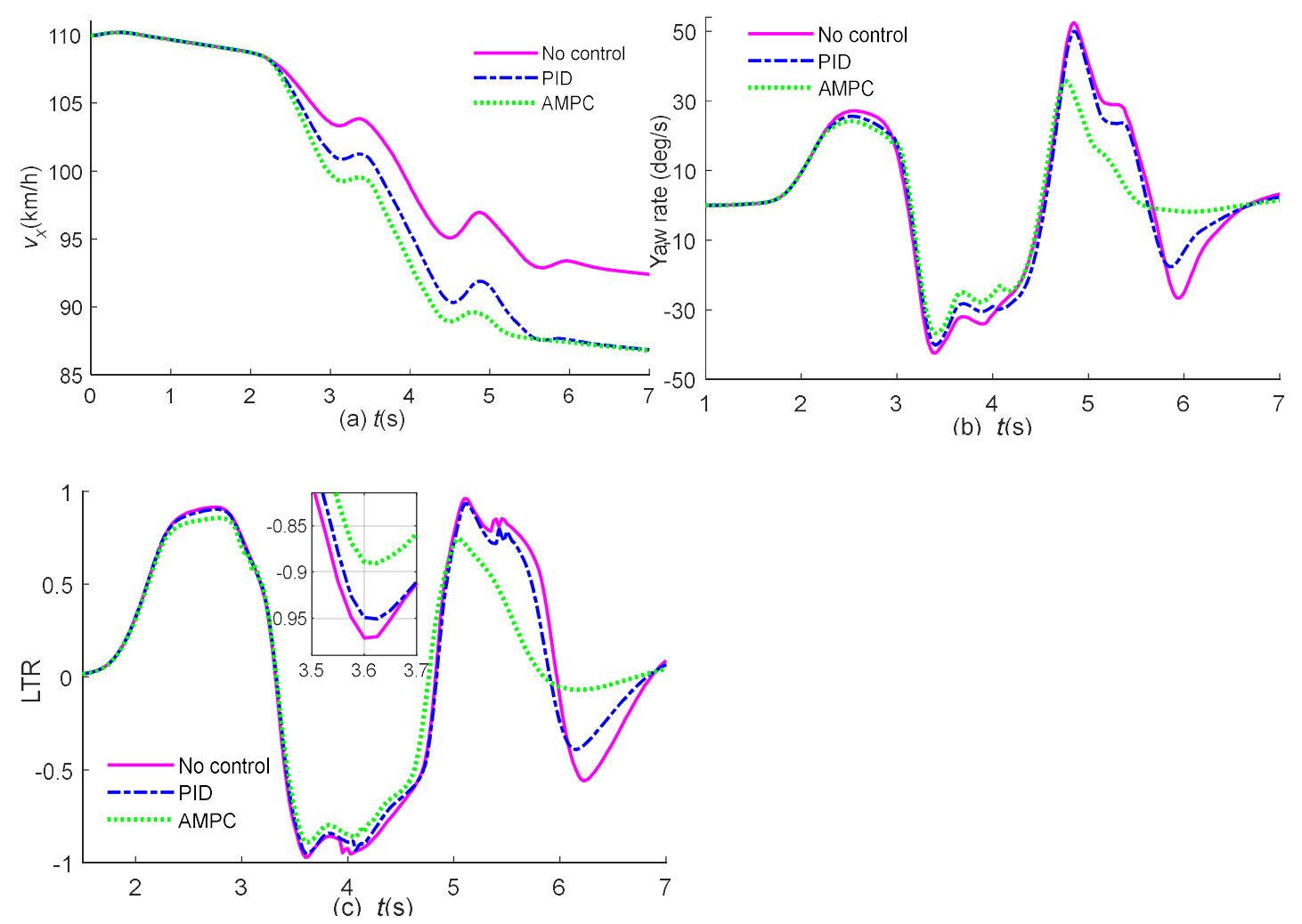

Figure 21. Stability indexes comparisons by different controllers: (a) Vehicle speed; (b) Yaw rate; (c) LTR.

Note that in Figure 21 (b) and (c) that the yaw rate peak values and LTR are decreased in the case of anti-rollover control vehicle. Whereas, the peak values of LTR by AMPC, PID, and no control are about $0.88,0.94$, and 0.98 respectively, which means the proposed rollover controller can enhance the roll stability of vehicle in emergency collision avoidance. Figure 22 shows AMPC control inputs of differential braking.

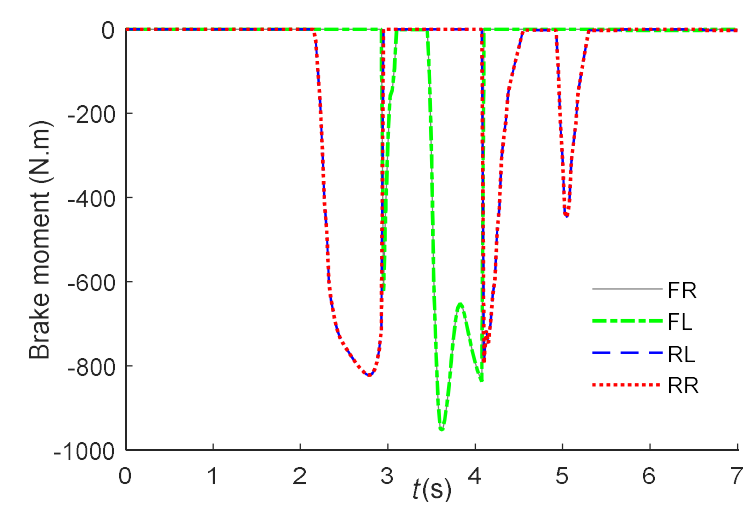

Figure 22. Active braking torque of 4 wheels by AMPC. 
In Figure 22, the rollover controller by AMPC generates a braking torque of 960 $\mathrm{N} \cdot \mathrm{m}$ to prevent rollover occurrence at $3.6 \mathrm{~s}$, and the vehicle speed also decreases rapidly which can prove that the braking controller has come into play.

\section{Integrated obstacle avoidance control}

The supervisor of the integrated obstacle avoidance control is as follows: According to the vehicle status signal, the yaw rate $\gamma$ and rollover index LTR are obtained. Then, they are compared with the $\gamma_{\mathrm{s}}$ (ideal yaw rate) and rollover threshold value $L T R_{\mathrm{s}}$. If the deviation of the actual and the ideal yaw rate is less than $\Delta \gamma_{\mathrm{s}}$, the active rear steering controller is not working, or the ARS is working. When the actual LTR is over LTRs, the $\mathrm{RBC}$ is open. The control strategy of the supervisory decision model is shown in Table 2.

Table 2. Control strategy of the supervisory decision.

\begin{tabular}{cllll}
\hline Control model & Selection conditions & 4WS & ARS & RBC \\
\hline 1 & $\left(\Delta \gamma<\Delta \gamma_{\mathrm{s}}\right)$ and $\left(\mathrm{LTR}<\mathrm{LTR}_{\mathrm{s}}\right)$ & Open & Close & Close \\
2 & $\left(\Delta \gamma>\Delta \gamma_{\mathrm{s}}\right)$ and $\left(\mathrm{LTR}<\mathrm{LTR}_{\mathrm{s}}\right)$ & Open & Open & Close \\
3 & $\left(\Delta \gamma>\Delta \gamma_{\mathrm{s}}\right)$ and $\left(\mathrm{LTR}>\mathrm{LTR}_{\mathrm{s}}\right)$ & Open & Open & Open \\
\hline
\end{tabular}

4WS: four-wheel steering; ARS: active rear steering; RBC: rollover braking control.

To verify the effectiveness of the integrated obstacle avoidance control $(\mathrm{ARS}+\mathrm{RBC})$ based on AMPC for highway obstacle avoidance, the dynamic visualization of obstacle avoidance is the same as Figure 20. The vs group are the ARS, RBC and no controlled vehicle. Figure 23 illustrates the path tracking performance by different controllers. Figure 24 shows the driving stability response of yaw and roll for emergency obstacle avoidance. 


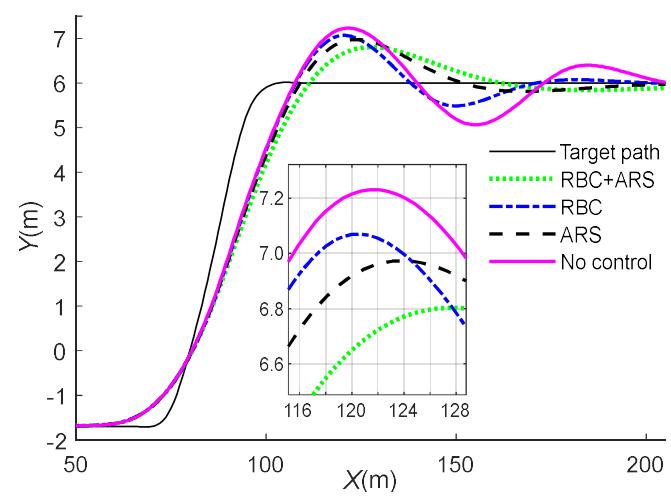

Figure 23. Vehicle tracking performance comparisons in emergency collision avoidance by different controllers.
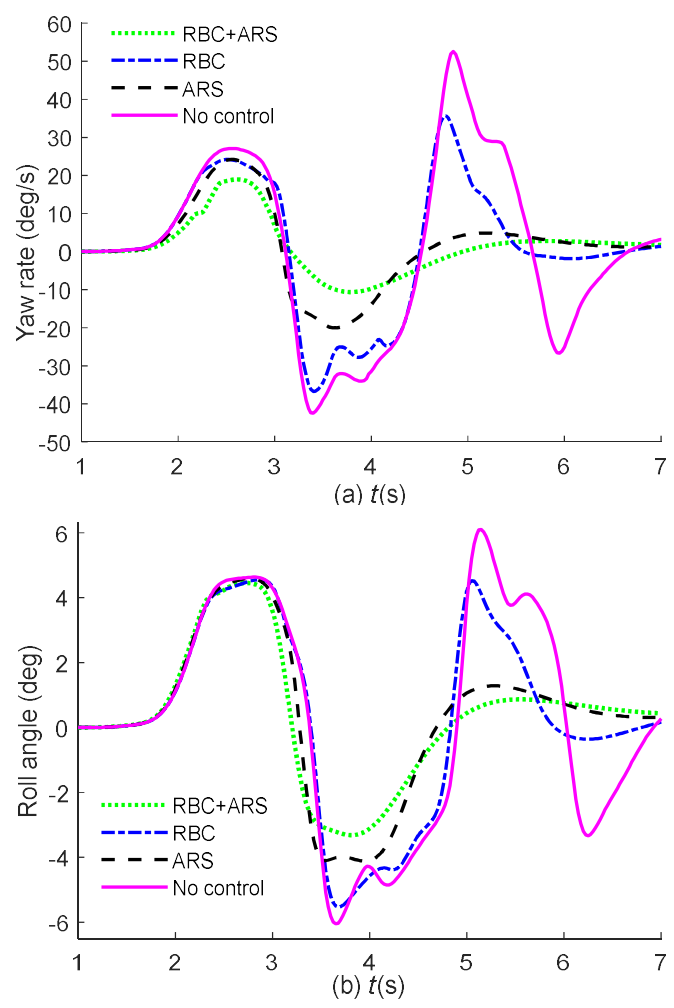

Figure 24. Vehicle driving stability indexes in emergency collision avoidance by different controllers: (a)Yaw rate; (b) Roll angle.

It implies from Figure 23 and Figure 24(a) that the no-controlled vehicle's yaw rate comes up to $54 \mathrm{deg} / \mathrm{s}$, it means the vehicle is close to lose lateral stability. But, the controlled vehicle by $\mathrm{RBC}+\mathrm{ARS}$ and ARS can maintain lateral stability. In addition, the tracking performance is also improved.

In Figure 24(b), the peak value of vehicle roll angle reduced more than $40 \%$ in case of RBC+ARS and $10 \%$ in case of RBC compared with the regular vehicle at $3.7 \mathrm{~s}$, 
in other words, the integrated controller can prevent rollover under emergency effectively. Consequently, the RBC+ARS vehicle can adjust its driving states of steering angle and lateral acceleration to perform steering and braking maneuvers by the AMPC.

In summary, the proposed integrated collision avoidance strategy based on APMC can coordinate the tracking accuracy and driving safety, and can prevent rollover in emergency.

\section{Conclusions}

An integrated collision avoidance strategy based on AMPC is proposed in this paper, which can not only guarantee the path tracking accuracy of the vehicle but also enhance the driving stability under emergency. The control architecture of the proposed novel active system consists of a supervisor and an adaptive MPC. The supervisory module decides the corresponding mode based on the critical safety threshold and ADAS sensors signal. The executive controller of $4 \mathrm{WS}+\mathrm{ARS}$ based on AMPC is used to carry out the supervisory module's requirements. To enhance the roll stability of intelligent vehicle in highspeed obstacle avoidance, RBC is beginning to work as the LTR over the safety threshold. Finally, the effectiveness of proposed obstacle avoidance control strategy is validated by Carsim-Simulink co-simulation. The results are summarized as follows.

For ARS model with a high weight on lateral displacement, the steering angle of rear wheels and yaw rate responses are larger than the controller with low weight, and ARS with a high weight of yaw rate index can obtain a better stability control performance.

The designed ARS and RBC work alone have limited effect on obstacle avoidance tracking characteristic and driving stability in emergency. 
The active obstacle avoidance strategy integrated with ARS, and RBC based on APMC can coordinate the tracking accuracy and driving safety, and can prevent rollover in emergency.

\section{Funding}

This work was supported in part by the Basic Research and Frontier Technology of the Chongqing Science and Technology Commission (grant no. cstc2020jcyj-msxmX0915), the National Natural Science Foundation of China (grant no. U1964202, 61773082).

\section{Author Contribution}

Conceptualization, H.L.; methodology and writing — original draft preparation, H.L.; data simulation, Z.G., J.L.; writing-review and editing, T. Z, Y. L.; project administration, Z.G., J. L.; supervision, J.L., H.L.; funding acquisition, H.L, Y. L. All authors have read and agreed to the published version of the manuscript.

Acknowledgments: The author greatly appreciated the financial support.

Conflicts of Interest: The authors declare no conflict of interest.

\section{References}

1. Chen Y., Chen S., Ren H., Gao, Z., \& Liu, Z. (2020). Path tracking and handling stability control strategy with collision avoidance for the autonomous vehicle under extreme conditions, IEEE Transactions on Vehicular Technology, 69(12), 14602 - 14617.

2. Guo, J., Ping, H., \& Wang, R. (2016). Nonlinear coordinated steering and braking control of vision-based autonomous vehicles in emergency obstacle avoidance. IEEE Transactions on Intelligent Transportation Systems, 17, (11),3230-3240.

3. Kuwata, Y., Karaman, S., Teo, J., Frazzoli, E., How, J. P., \& Fiore, G. (2009). Real-time motion planning with applications to autonomous urban driving. IEEE Transactions on Control Systems Technology, 17(5), 1105-1118.

4. Wang C.Y., Zhao W.Z., Xu Z.J., \& Zhou G. (2017). Path planning and stability control of collision avoidance system based on active front steering, Science China Technological Sciences, 60(8),1231-1243. 
5. Claussmann L., Revilloud M., Gruyer D., Glaser S. (2020). A review of motion planning for highway autonomous driving, IEEE Transactions on Intelligent Transportation Systems, 21, (5), 1826 - 1848.

6. Klier, W., Reimann, G., \& Reinelt, W. (2004). Concept and functionality of the active front steering system. SAE technical paper, 2004-21-0073.

7. Hu, C., Zhao, L., Cao, L., Tjan, P., \& Wang, N. (2020). Steering control based on model predictive control for obstacle avoidance of unmanned ground vehicle. Measurement and Control, 53(5), 002029401987887.

8. Yim S. (2020). Comparison among active front, front independent, 4-wheel and 4-wheel independent steering systems for vehicle stability control, Electronics, 9 (5), 798.

9. Hajiloo, R., Abroshan, M., Khajepour, A., Kasaiezadeh, A., \& Chen, S. K. (2021). Integrated steering and differential braking for emergency collision avoidance in autonomous vehicles. IEEE Transactions on Intelligent Transportation Systems, 22(5), 3167 - 3178.

10. Jan, L. (2019). Adaptive cruise control with guaranteed collision avoidance. IEEE Transactions on Intelligent Transportation Systems, 20(5),1897-1907.

11. Glaser, S., Vanholme, B., Mammar, S., Gruyer D., \& Nouveliere, L. (2010). Maneuver-based trajectory planning for highly autonomous vehicles on real road with traffic and driver interaction. IEEE Transactions on Intelligent Transportation Systems, 11(3), 589-606.

12. Llorca, D. F., Milanes, V., Alonso, P. I., Gavilan, M., Daza I. G., Perez J., \& Sotelo M. A. (2011). Autonomous pedestrian collision avoidance using a fuzzy steering controller. IEEE transactions on intelligent transportation systems. 12(2), 390-401.

13. Schildbach, G., \& Borrelli, F. (2015). Scenario model predictive control for lane change assistance on highways. IEEE Intelligent Vehicles Symposium. (IV), 611-616.

14. Pourasad, Y., Mahmoodi-K, M., \& Oveisi, M. (2016). Design of an optimal active stabilizer mechanism for enhancing vehicle rolling resistance. Journal of Central South University, 23(5), 1142-1151.

15. Milakis, D., Arem, B. V, \& Wee, B. V. (2017). Policy and society related implications of automated driving: a review of literature and directions for future research. Journal of Intelligent Transportation Systems Technology Planning \& Operations, 21(4), 324-348.

16. Yang, X., Wang, Z., \& Peng, W. (2009). Coordinated control of AFS and DYC for vehicle handling and stability based on optimal guaranteed cost theory. Vehicle System Dynamics, 47(1), 57-79.

17. Wu, J., Wang, X. G., Li, L., Qin, C. A., \& Du, Y. C. (2018). Hierarchical control strategy with battery aging consideration for hybrid electric vehicle regenerative braking control. Energy, 145, 301-312. 
18. Ghazali, M., Durali, M., \& Salarieh, H. (2017). Vehicle trajectory challenge in predictive active steering rollover prevention. International Journal of Automotive Technology, 18(3), $511-521$.

19. Li, H., Zhao, Y., Lin, F., \& Zhu, M. (2018). Nonlinear dynamics modeling and rollover control of an off-road vehicle with mechanical elastic wheel. Journal of the Brazilian Society of Mechanical Sciences \& Engineering, 40(2), 51.

20. Li, L., Lu, Y., Wang, R., \& Chen, J. (2017). A three-dimensional dynamics control framework of vehicle lateral stability and rollover prevention via active braking with MPC., IEEE Transactions on Industrial Electronics, 64(4), 3389-3401.

21. Rosolia, U., Bruyne, S. D., \& Alleyne, A. G. (2017). Autonomous vehicle control: a nonconvex approach for obstacle avoidance. IEEE Transactions on Control Systems Technology, 25(2), 469-484.

22. Li, H. G., Zhao, Y. Q., Lin, F., \& Xiao, Z. (2019). Integrated yaw and rollover control based on differential braking for off-road vehicles with mechanical elastic wheel. Journal of Central South University, 26(9), 2354-2367.

23. Li, H., Li, J., Su, Z., Wang, X., \& Luo, J. (2020). Research on active obstacle avoidance control strategy for intelligent vehicle based on active safety collaborative control. IEEE Access, (8), 183736 - 183748.

24. Cui, Q., Ding, R., Wu, X., \& Zhou, B. (2020). A new strategy for rear-end collision avoidance via autonomous steering and differential braking in highway driving. Vehicle System Dynamics, 58(6), 955-986.

25. Qian, X., Wang, C., \& Zhao, W. (2020). Rollover prevention and path following control of integrated steering and braking systems. Proceedings of the Institution of Mechanical Engineers Part D Journal of Automobile Engineering, 234(6), 1644-1659.

26. Song, J., \& Che, W. S. (2009). Comparison between braking and steering yaw moment controllers considering abs control aspects. Mechatronics, 19(7), 1126-1133. 